\title{
A review on processing factors of pesticide residues during fruits processing
}

\author{
Moo-Hyeog $\mathbf{I m}^{1}$ (D) $\cdot$ Yu-Jeong $\mathbf{J i}^{2}$ \\ 과일류 가공 중 잔류농약 가공계수에 관한 고찰
}

임무혁 ${ }^{1}$ - 지유정

Received: 16 March 2016 / Accepted: 9 May 2016 / Published Online: 30 September 2016

(C) The Korean Society for Applied Biological Chemistry 2016

\begin{abstract}
A processing factor (PF) has been used to define the maximum residue limits of pesticide in a variety of processed fruit products. This study summarizes PF by the stage of fruit processing based on JMPR reports from 2010 to 2014. When we compared $\mathrm{PF}$ of 19 pesticides in apple products during the processing of washing, drying, canning, juice, sauce, puree and pomace, PF was higher than 1 only in pomace. In the comparison of 21 pesticides during the process of grape (washing, juice, wine and raisin), PF was higher than 1 in raisin. In the comparison of 19 pesticides during the process of orange (juice, oil and marmalade), PF was higher than 1 in oil. When 27 pesticides were compared during the process of tomato (juice, puree and paste), paste showed the highest PF value except pomace. During the process of plum (washing, drying and puree) with 12 pesticides, PF was higher than 1 in drying. The correlation coefficients between physical characteristics of pesticide (fat-solubility and volatility) and PF were statistically significant in the processes of apple juice, orange juice, tomato puree and paste and strawberry jam.
\end{abstract}

Moo-Hyeog $\operatorname{Im}(\bowtie)$

E-mail:imh0119@daegu.ac.kr

${ }^{1}$ Department of Food Engineering, Daegu University, Gyeongbuk 38453, Republic of Korea

${ }^{2}$ Department of Food Engineering, Daegu University, Gyeongbuk 38453, Republic of Korea

This is an Open Access article distributed under the terms of the Creative Commons Attribution Non-Commercial License (http://creativecommons. org/licenses/by-nc/3.0/) which permits unrestricted non-commercial use, distribution, and reproduction in any medium, provided the original work is properly cited.
Keywords CODEX - fruit processing - Joint Meeting of the FAO Panel of Experts on Pesticide Residues · pesticide residue · processing factor

\section{서 론}

농약은 농작물에 해를 끼치는 병 - 해충을 방제하여 농산물의 양적 증산과 품질을 향상시켜 먹거리를 풍요롭게 할 수 있다. 그러나, 농약을 잘못 사용함으로써 생산된 일부 농산물에서 잔 류량이 허용기준을 초과하기도 한다. 그 결과로 국민건강과 관 련하여 농업인에게는 중독피해를 주기도 하고, 소비자에게는 막 연한 불안감을 느끼게 할 수도 있다. 국제식품규격위원회 (CODEX) 및 세계 각국에서는 식품 중 잔류농약 안전관리를 위 하여 각 농산물별 또는 농산물 그룹별로 사람이 일생을 걸쳐 매일 섭취하여도 인체에 아무런 해를 주지 않는 수준의 농약잔 류허용기준(Maximum Residue Limit, MRL)을 설정하여 안전한 식품이 유통될 수 있도록 하고 있다(Lee와 Woo 2010; Chung 등, 2011; FAO와 WHO 2015). 농약 개발회사, 판매회사 및 수입회사 등에서 $\mathrm{CODEX}$ 또는 각국 정부에 식품 중 농약잔류 허용기준 설정을 요청하기 위해서는 농약 성분에 대한 일일섭 취허용량(Acceptable Daily Intake, ADI) 설정을 위한 급성, 아 급성, 아만성, 만성 독성자료, 농작물을 재배하면서 농약을 살 포한 농산물에 대한 농약잔류성적 및 식품의 가공 중 농약 감 소계수 자료 등을 제출하여야 된다. 이러한 자료가 제출-평가 되어 식품 중 농약의 $\mathrm{MRL}$ 이 설정된 후에 해당 농작물에 대한 농약으로 사용될 수 있게 된다(MFDS 2015b).

식품 중 농약의 $\mathrm{MRL}$ 설정을 위해서 제안된 기준(안)과 국 민 평균 식품섭취량을 활용하여 노출평가를 수행하게 된다 (MFDS 2015b). 일반적으로 세계각국 및 CODEX에서는 가공 
된 식품이 아닌 원료 상태의 식품에 대하여 농약잔류허용기준 을 설정하고 있다. 원료식품에 잔류된 농약은 식품조리 및 가 공과정 중 세척, 열분해 및 휘발 등으로 대부분 감소될 것으로 예상되지만, 건조나 농축 과정 중에는 원료식품에 잔류된 양보 다 증가되는 경우도 발생할 수 있다. 국제식품규격위원회 농약 전문가위원회(Joint meeting $\mathrm{FAO} / \mathrm{WHO}$ on Pesticides Residues, $\mathrm{JMPR}$ )에서는 원료식품에 잔류된 농약이 가공식품으로 가공된 후 농축될 경우 별도의 기준을 설정하고, 가공 후 감소될 경우 에는 노출평가에 활용하고 있다(FAO 2009). 우리나라에서는 가 공 후 농약이 농축될 우려가 있는 식품에 대한 연구사업을 통 하여 인삼, 밀가루 및 건조농산물 등의 가공식품에 별도의 $\mathrm{MRL}$ 을 설정하고 있다 $(\mathrm{Im}$ 등, 2006; Im 등, 2007; Kim 등, 2009; Lee 등, 2009; Park 등, 2009a, b; Lee 등, 2012; Noh 등, $2012 a, b)$. 원료식품에는 농약기준이 있으나, 가공식품에는 별도 의 농약기준이 없을 경우, 원료에 허용된 농약기준을 환산하여 그대로 가공식품에 적용하고 있는 실정이다(MFDS 2015a). 원 료 중에 잔류된 농약은 가공과정 중 제거 또는 감소되거나 농 축될 수 있어서, 원료식품에 설정된 MRL을 그대로 가공식품에 적용할 경우 불합리할 수도 있다. 식품 중 농약 MRL 설정 시, 원료에서 가공식품으로 가공된 후 농약의 잔류양상을 파악하여 섭취량평가를 수행하는 것이 이상적일 것이다.

$\mathrm{CODEX}$ 에서는 각국 또는 농약회사에서 제출된 과일류 가공 식품에 대한 연구자료를 평가하여 세척, 껍질 벗기기, 주스, 오 일, 건조, 퓌레 및 페이스트 등에 대한 잔류농약 가공계수 자료 를 $\mathrm{JMPR}$ 에서 평가하고 필요한 경우 별도로 가공식품에 대한 기준을 설정하고 있다. 사과 가공(Athanasopoulos와 Pappas, 2000; Zabik 등, 2000; Li 등, 2015), 건포도 가공(Cabras 등, 1998b; Lentza-Rizos 등, 2006), 자두 및 살구 건조(Cabras 등, 1998a, c), 복숭아 통조림 가공(Lentza-Rizos 1995), 토마토 가 공(Han 등, 2013) 등 과일류의 전처리 및 가공 중 농약잔류량 의 변화에 대한 연구가 보고되고 있다. Keikotlhaile 등(2010)은 식품가공 중 농약감소에 관한 여러 연구자료로 메타분석(metaanalysis)을 통하여 잔류농약의 감소 또는 농축에 대한 기여도 평가를 하였고, Kaushik 등(2009)은 식품의 가공방법 별 농약 감소방안 마련을 위하여 여러 연구를 고찰하였다. 그러나 과일 류의 가공 후 농약잔류허용기준 설정 필요성을 추정하기 위하 여 농약의 물리화학적 특성 등에 따른 농약의 감소 또는 농축 경향에 대하여 다양한 자료를 고찰한 연구는 거의 없는 실정이 다. 과일류 가공식품에 대한 농약잔류허용기준설정을 위하여 필 요한 자료 생산을 위해서 많은 시간과 비용이 소요되므로 자료 생산 이전에 다양한 실험결과를 고찰하여 사전에 가공 후 잔류 량 변화를 예측할 수 있는 모델이 있을 경우 이러한 어려움을 해결할 수 있을 것이다. 식품의 가공 방식은 각 국가별 식이 섭 취패턴에 따라 매우 다양하므로 MRL 설정 시 식품 가공과정 중 잔류농약 변화 모델이 있을 경우도 많은 도움이 될 것이다. $\mathrm{JMPR}$ 에서 평가한 과일류 가공식품에 대한 농약 잔류량 변화자 료는 비교적 일관성 있는 자료이므로 본 연구에서는 식품의 가 공방법 별 농약 가공계수를 조사하여 농약 물리화학적 특성과 의 상관성을 고려하여 향후 과일류 가공에 따른 농약 기준설정 자료 생산 시 예측모델로 활용할 수 있도록 하고자 하였다.

\section{재료 및 방법}

\section{식품 가공 중 농약 가공계수 자료}

$\mathrm{CODEX}$ 에 농약잔류허용기준 설정을 위하여 제출된 자료는 가 이드라인에 제시된 권고사항에 따라 가능한 단순한 가공처리와 제시된 일관성 있는 온도 및 시간에 따라 연구되었다(FAO 2009). 본 연구에서는 가공식품 중 잔류농약 가공 연구자료를 $\mathrm{CODEX}$ 농약잔류허용기준 설정을 위하여 JMPR에서 평가한 결 과보고서 중 2010-2014년 자료를 활용하였다. 식품 중 잔류농 약의 가공계수(processing factor, PF)는 원료의 농약 잔류량에 대한 가공 후 식품에 잔류하는 농약 잔류량의 비율이다.

$$
\begin{aligned}
& \text { Processing Factor (PF) } \\
& =\frac{\text { Residue level in processed food }}{\text { Residue level in raw agricultural food }}
\end{aligned}
$$

\section{가공 방법 및 잔류농약 분석 방법}

Table 1에는 과일류의 가공에 사용된 일반적인 방법에 대하여 요약하였다. 주스의 경우에는 일반적인 주스 가공방법 및 사과 와 포도 주스에 효소처리를 하는 방법으로 정리하였으며, 과일 건조는 일반적인 방법을 정리하였다. 통조림은 사과와 토마토를 구분하였으며, 오일은 오렌지 오일 제조법을 요약하였다. 그 외 오렌지 마멀레이드, 건포도, 포도주, 토마토 케첩, 퓌레, 페이스 트, 소스 및 과일 잼 제조방법을 요약하였다. Table 2에는 $\mathrm{JMPR}$ 에서 평가한 결과보고서에서 각 농약 성분 별로 가공된 식품 중 잔류농약 분석 시 전처리에 이용된 추출용매와 HPLC, $\mathrm{GC}, \mathrm{MS}$, 및 $\mathrm{MS} / \mathrm{MS}$ 등의 분석기기에 대한 내용을 요약하였다.

\section{자료 분석 방법}

$\mathrm{JMPR}$ 에서 평가된 사과(19종 농약) (세척, 건조, 통조림, 주스, 소스, 퓌레 및 pomace), 포도(21종 농약) (세척, 주스, 포도주, 건포도 및 pomace), 오렌지(19종 농약) (껍질, 주스, 오일, 마멀 레이드, molasses 및 pomace), 토마토(27종 농약) (세척, 껍질, 통조림, 주스, 퓌레, 페이스트, 케첩 및 pomace), 자두(12종 농 약) (세척, 건조 및 퓌레), 딸기(7종 농약), 블루베리(1종 농약), 체리(3종 농약), 망고(1종 농약), 파인애플(1종 농약)의 세척, 통 조림, 데치기, 건조, 주스 및 잼 가공 중 농약의 가공계수 자료 를 조사하여 비교하였다. 물/옥탄올 분배계수 $\left(\mathrm{K}_{\mathrm{ow}}\right)$ 는 농약의 지 용성/수용성을 나타내며 증기압은 휘발성의 지표이다. JMPR에 주로 제출되는 유제(EC), 액상수화제(SC) 및 기타와 같은 각 농 약의 제형과 $\mathrm{K}_{\mathrm{ow}}$ 의 상관관계를 엑셀의 상관분석을 이용하여 비 교하고 자유도에 따라 유의성을 결정하였다. 증기압과 침투성 및 비침투성으로 분류되는 작용특성에 따른 상관관계도 같은 방 법으로 결정하였다.

\section{결과 및 고찰}

사과의 잔류농약 가공계수

$\mathrm{CODEX}$ 에 사과 농약잔류허용기준 설정을 위하여 제출된 19종 
Table 1 General processing methods of Fruits

\begin{tabular}{|c|c|}
\hline Commodity & Processing method \\
\hline Juice & $\begin{array}{l}\text { - Washing - crushing - filtering (pressing) - sterilization (general method) } \\
\text { - Washing - crushing - heating - enzyme treatment - filtering (pressing) - sterilization - juice and pomace (apple, grape) } \\
\text { - Washing - chopping - blanching - filtering - sterilization - juice and pomace (tomato) }\end{array}$ \\
\hline Dry & - Washing - slicing - drying at $60-70{ }^{\circ} \mathrm{C}$ \\
\hline Can & $\begin{array}{l}\text { - Peeling - slicing - coring - seaming - sterilization (apple) } \\
\text { - Washing - removing the skins - seaming - sterilization (tomato) }\end{array}$ \\
\hline Oil & - Washing - crushing - centrifugation (orange) \\
\hline Marmalade & - Washing - slicing peel + mincing fruit - cooking - addition of sugar - heating (orange) \\
\hline Raisin & - Washing - sundried (or sundried - dried at $55-60^{\circ} \mathrm{C}$ ) \\
\hline Wine & - Washing - crushing - fermentation - filtering - store (grape) \\
\hline Ketchup & - Raw tomato juice - evaporation - addition of sugar, vinegar and salt - sterilization (tomato) \\
\hline Puree & - Raw juice - evaporation $\left(12-12.5^{\circ}\right.$ Brix $)$ - sterilization \\
\hline Paste & - Raw juice - evaporation $\left(20-30^{\circ}\right.$ Brix $)$ - sterilization \\
\hline Sauce & $\begin{array}{l}\text { - Peeling - coring - slicing - mashing - heating - evaporation (apple 1) } \\
\text { - Washing - cutting - blanching - sieving - addition of sugar - canning - sterilization (apple 2) }\end{array}$ \\
\hline Jam & - Washing - addition of sugar, water and pectin - boiling \\
\hline
\end{tabular}

농약 자료 중 $\mathrm{PF}$ 연구자료를 가공방법 별로 정리하여 비교한 결과를 Table 3에 나타내었다. 세척 후 $0.55-0.97$ (평균 $0.68 \pm 0.17$ ), 건조 후에는 0.1-0.64(평균 $0.44 \pm 0.21$ ), 통조림은 불검출-0.22(평 균 $0.08 \pm 0.09$ ), 주스로 가공한 후에는 불검출- 0.5 (평균 $0.16 \pm 0.16$ ), 사과 소스는 불검출-2.7(평균 $0.67 \pm 0.90$ ), 퓌레는 0.3-1.0(평균 $0.49 \pm 0.34$ ) 및 pomace (wet)는 0.24-9.5(평균 3.1 \pm 2.5 )로 조사 되었다. 사과 가공 방법에 따른 농약의 가공계수를 비교한 결 과, 사과를 물로 세척할 경우 $\mathrm{PF}$ 가 평균 0.68 로 약 $32 \%$ 정도 의 농약성분이 제거됨을 알 수 있었다. 사과의 건조 가공에서 는 사과를 세척, 박피 및 절단 한 후, $60-70^{\circ} \mathrm{C}$ 에서 장시간 열 풍 건조로 인하여 $80 \%$ 이상의 수분이 증발되어 $10 \%$ 정도의 수분함량까지 건조되어 농축되었음에도 불구하고 $\mathrm{PF}$ 는 평균 0.44 로 나타났다. 세척 및 박피 과정에서 농약 성분이 감소되고 열풍 건조 과정 중 열에 의해 잔류농약이 상당량 분해됨을 알 수 있었다. 통조림 가공계수가 평균 0.08 로 가공법 중 농약이 가장 많이 감소되었다. 이 결과는 Table 1의 통조림 가공 방법 에서와 같이 가공 시 사과 껍질의 제거 및 통조림의 품질과 저 장성에 중요한 영향을 미치는 살균공정이 농약 감소에 큰 역할 을 한다고 판단된다. 사과를 주스로 가공한 경우에는 $\mathrm{PF}$ 가 평 균 0.16 으로 조사되었다. Table 3 의 사과에 사용된 19종 농약의 $\mathrm{K}_{\mathrm{ow}}$ 값이 0.893-6.9로 지용성 특성을 가지고 있어서 세척, 분쇄, 효소처리, 여과 및 살균 과정을 거친 후 농약 성분은 pomace 에 대부분 잔류되고 주스에는 평균 $16 \%$ 정도만 잔류된 것으 로 판단된다. 사과 소스 제조는 사과를 박피, 제심, 파쇄, 가열 및 농축한 후 설탕을 첨가한 방법과 세척, 절단, 데치기, 여과, 설탕 첨가 및 캔으로 제조하여 살균하는 두 가지 방법이 있다. 소스 제조 시 농축 과정이 포함된 cyantraniliprole과 cyflumetofen 의 경우에는 $\mathrm{PF}$ 가 2.2 와 2.7로 나타났으나, 농축 과정이 없는 다른 농약 성분들의 $\mathrm{PF}$ 는 불검출- 0.6 으로 상당량의 농약 성분 이 감소되었다. 퓌레는 주스를 제조한 후 농축과정을 거쳐 최 종 제품으로 가공되는데, $\mathrm{PF}$ 가 0.49 로 나타나 가공 중 상당량
의 농약이 감소됨을 알 수 있었다. 사과의 가공 공정 중 19 종 농약의 $\mathrm{PF}$ 연구자료를 평가한 결과, 소스 가공을 제외한 대부 분의 가공 공정에서 $\mathrm{PF}$ 값이 1 이하로 조사되어서 잔류된 농 약 성분은 가공 중 상당량이 감소됨을 알 수 있었다. 사과 pomace는 주스 제조 시 부산물로 생산되는 것으로 농약 성분 의 상당량이 농축되었으나, 대부분 사료로 사용되므로 사료에 대한 기준 설정 시 $\mathrm{PF}$ 값을 고려할 수 있을 것이다. Zabik 등 (2000)에 의한 사과의 소스 및 주스 가공 중 azinphos-methyl, chlorpyrifos, esfenvalerate 및 methomyl 연구 결과에는 소스에 서 불검출- $94.1 \%$ 및 주스에서 불검출-90\% 감소되는 결과로 대 부분의 처리구에서 잔류농약이 검출되지 않아서 비슷한 경향이 지만, 본 연구에 사용된 자료들의 결과보다 가공 중 상당량의 잔류농약이 감소되었다. $\mathrm{Li}$ 등(2015)에 의한 cypermethrin, chlorpyrifos, tebuconazole, acetamiprid 및 carbendazim의 사과 세척 시 가공계수는 0.34-0.94, 주스 가공 시에는 0.17-0.91로 본 연구에 사용된 자료의 결과와 비슷한 경향을 보였다.

Table 4에서는 농약의 물리화학적 특성 중 $\mathrm{K}_{\mathrm{ow}}$ 및 증기압에 대한 사과 가공 방법 간의 상관관계를 나타내었다. $\mathrm{K}_{\mathrm{ow}}$ 및 주 스 $\mathrm{PF}$ 사이에는 유의한 부상관 관계가 있었다. $\mathrm{K}_{\mathrm{ow}}$ 값이 높아 질수록 농약 성분이 주스로 이행될 확률이 낮음을 알 수 있었 다. 특히 $\mathrm{EC}$ 및 $\mathrm{SC}$ 제형이 살포된 사과 주스의 $\mathrm{PF}$ 는 $\mathrm{K}_{\mathrm{ow}}$ 값 과 유의한 부상관 관계를 보였다. 가공방법에 따른 $\mathrm{K}_{\mathrm{ow}}$ 및 증 기압간에는 주스를 제외한 대부분의 가공과정 중 상관관계가 무 의미한 낮은 상관 관계가 나타났다.

\section{포도의 잔류농약 가공계수}

Table 5에 포도에 대한 21종 농약의 세척, 주스, 포도주, 건포 도 및 pomace PF 연구자료를 가공방법 별로 정리 - 비교하였 다. 포도 주스 가공 시 $\mathrm{PF}$ 값은 불검출- 1.7 (평균 $0.50 \pm 0.55$ )로 매우 다양한 결과로 조사되었다. 주스 가공계수 중 clothianidin 이 1.45 , dichlobenil 1.4, dinotefuran 1.2 및 etoxazole 1.7 로 
Table 2 Analytical methods of pesticides for processed foods

\begin{tabular}{|c|c|c|c|c|c|c|c|c|c|}
\hline Pesticide & Commodity & Extraction solvents & Analysis Instrument & Reference & Pesticide & Commodity & Extraction solvents & Analysis Instrument & Reference \\
\hline \multirow[t]{3}{*}{ Ametoctradin } & Grape & Methanol:water & HPLC-MS/MS & JMPR 2012a & Fludioxonil & Pineapple & Methanol:water & GC-MS & JMPR $2013 \mathrm{f}$ \\
\hline & & & & & & Tomato & Methanol & HPLC & JMPR $2012 f$ \\
\hline & Tomato & Methanol:water & HPLC-MS/MS & & Fluensulfone & Tomato & Acetonitrile & HPLC & JMPR 2014f \\
\hline Bifenthrin & Tomato & Acetonitrile & GC-MS & JMPR 2010a & Fluopyram & Apple & Acetonitrile:water & HPLC-MS/MS & JMPR 2010i \\
\hline Boscalid & Orange & Methanol:water:HCI & HPLC-MS/MS & JMPR 2010b & & Grape & Acetonitrile:water & HPLC-MS/MS & JMPR 2012g \\
\hline Chlorantraniliprole & Orange & Acetonitrile:water & GC-ECD & JMPR 2010c & & Orange & Acetonitrile:water & HPLC-MS/MS & \\
\hline Chlorfenapyr & Tomato & Methanol:water & GC-ECD & JMPR 2012b & & Strawberry & Acetonitrile:water & HPLC-MS/MS & \\
\hline Chlorothalonil & Grape & Acetone:sulphuric acid & GC-MS & JMPR 2010d & & Tomato & water & HPLC-MS/MS & \\
\hline \multirow[t]{3}{*}{ Clothianidin } & Apple & Acetone:water & HPLC-UVD & JMPR 2010e & Fluopyram & Blueberry & Acetonitrile:water & HPLC-MS/MS & JMPR 2014g \\
\hline & Grape & Methanol:water & HPLC-UVD & & & Plum & Acetonitrile:water & HPLC-MS/MS & \\
\hline & Tomato & Methanol:water & HPLC-UVD & & Flutriafol & Apple & Acetonitrile:water & HPLC-MS/MS & JMPR $2011 \mathrm{c}$ \\
\hline \multirow[t]{5}{*}{ Cyantraniliprole } & Apple & Acetonitrile & HPLC-MS/MS & JMPR 2013a & & Grape & Acetonitrile:water & HPLC-MS/MS & \\
\hline & Grape & Acetonitrile & HPLC-MS/MS & & Fluxapyroxad & Apple & Methanol:water:HCI & HPLC-MS/MS & JMPR $2012 \mathrm{~h}$ \\
\hline & Orange & Acetone & HPLC-MS/MS & & & Plum & Methanol:water:HCI & HPLC-MS/MS & \\
\hline & Plum & Cyantraniliprole & HPLC-MS/MS & & & Tomato & Methanol:water:HCI & HPLC-MS/MS & \\
\hline & Tomato & Acetonitrile & HPLC-MS/MS & & Glufosinate- & Orange & Water & GC-FPD & JMPR 2012i \\
\hline \multirow[t]{2}{*}{ Cycloxydim } & Strawberry & Isopropanol:water & HPLC-MS/MS & JMPR 2012c & & Plum & Water & HPLC-MS/MS & \\
\hline & Tomato & Isopropanol:water & HPLC-MS/MS & & Hexythiazox & Strawberry & Methanol & GC-ECD & JMPR 2011d \\
\hline \multirow[t]{4}{*}{ Cyflumetofen } & Apple & Acetonitrile:water & HPLC & JMPR 2014a & Methoxyfenozide & Orange & Methanol & HPLC-UVD & JMPR $2012 \mathrm{j}$ \\
\hline & Grape & Acetonitrile:water & HPLC-MS/MS & & Metrafenone & Grape & Methanol:water & HPLC-MS & JMPR $2014 \mathrm{~h}$ \\
\hline & Orange & Acetonitrile:water & HPLC-MS/MS & & Myclobutanil & Apple & Methanol & HPLC-MS/MS & JMPR 2014i \\
\hline & Tomato & Acetonitrile:water & HPLC-MS/MS & & & Grape & Methanol & HPLC-MS/MS & \\
\hline \multirow{3}{*}{$\begin{array}{c}\text { Cyfluthrin/ } \\
\text { beta-cyfluthrin }\end{array}$} & Apple & Acetone & GC-ECD & JMPR 2012d & & Tomato & Methanol & HPLC-MS/MS & \\
\hline & Orange & Acetone & GC-ECD & & Novaluron & Tomato & Methanol:water & GC-ECD & JMPR 2010j \\
\hline & Tomato & Acetone & GC-ECD & & Penthiopyrad & Apple & Acetonitrile:water & HPLC-MS/MS & JMPR 2012k \\
\hline \multirow[t]{2}{*}{ Cyprodinil } & Apple & Methanol:water & HPLC-MS/MS & JMPR 2013b & & Plum & Acetonitrile:water & HPLC-MS/MS & \\
\hline & Tomato & Methanol:water & HPLC-UVD & & & Tomato & Methanol:water & HPLC & \\
\hline Dichlobenil & Grape & Methanol & HPLC & JMPR 2014b & Propamocarb & Tomato & Acetonitrile & HPLC-MS/MS & JMPR 2014j \\
\hline
\end{tabular}




\begin{tabular}{|c|c|c|c|c|c|c|c|c|c|}
\hline Pesticide & Commodity & Extraction solvents & Analysis Instrument & Reference & Pesticide & Commodity & Extraction solvents & Analysis Instrument & Reference \\
\hline \multirow[t]{3}{*}{ Difenoconazole } & Apple & Methanol:water & GC-NPD & JMPR 2013c & Propiconazole & Orange & Methanol:water & HPLC-MS/MS & JMPR 2013g \\
\hline & Orange & Methanol:water & HPLC & & Pyraclostrobin & Cherry & Methanol:water:HCI & HPLC-MS/MS & JMPR 2011e \\
\hline & Tomato & Methanol:water & GC-NPD & & & Plum & Methanol:water:HCI & HPLC-MS/MS & \\
\hline Dimethomorph & Strawberry & Acetone:water & GC-NPD & JMPR 2014c & & Orange & Methanol:water & HPLC-MS/MS & \\
\hline \multirow[t]{2}{*}{ Dinotefuran } & Grape & Acetonitrile:water & HPLC-MS/MS & JMPR 2012e & & Strawberry & Methanol:water:HCI & HPLC-MS/MS & \\
\hline & Tomato & Acetonitrile:water & HPLC-MS/MS & & Pyrimethanil & Apple & Acetone & HPLC-UVD & JMPR 2013h \\
\hline \multirow[t]{3}{*}{ Dithianon } & Cherry & Acetone & GC-MS & JMPR 2013d & Sulfoxaflor & Apple & Acetonitrile:water & HPLC-MS/MS & JMPR $2011 \mathrm{f}$ \\
\hline & Grape & Acetonitrile:water: $\mathrm{HCl}$ & HPLC-MS/MS & & & Cherry & Acetonitrile:water & HPLC-MS/MS & \\
\hline & Plum & Acetonitrile:water: $\mathrm{HCl}$ & HPLC-MS/MS & & & Grape & Acetonitrile:water & HPLC-MS/MS & \\
\hline Emamectin benzoate & Apple & Methanol & HPLC-MS/MS & JMPR 2011a & & Orange & Acetonitrile:water & HPLC-MS/MS & \\
\hline \multirow[t]{2}{*}{ Etofenprox } & Apple & Dichloromethane:hexane & HPLC & JMPR 2011b & & Strawberry & Acetonitrile:water & HPLC-MS/MS & \\
\hline & Grape & Methanol:water & HPLC & & Tebuconazole & Apple & Acetone & GC-NPD & JMPR 2011g \\
\hline \multirow[t]{2}{*}{ Etoxazole } & Grape & Acetone & GC-MS/MS & JMPR $2010 \mathrm{f}$ & & Grape & Methanol:acetone & HPLC & \\
\hline & Orange & Acetone:water & GC-MS & & & Plum & Acetone & GC-NPD & \\
\hline Fenamidone & Grape & Acetone:water & GC-NPD & JMPR 2014d & & Tomato & Acetone:water & GC-MSD & \\
\hline Fenbuconazole & Orange & Methanol & GC-ECD & JMPR 2013e & Thiamethoxam & Mango & Methanol:water & HPLC-MS/MS & JMPR $2014 \mathrm{k}$ \\
\hline \multirow[t]{2}{*}{ Fenpropathrin } & Orange & Ethyl acetate:cyclohexane & GC-MS & JMPR $2014 \mathrm{e}$ & Tolfenpyrad & Orange & Methanol & HPLC & JMPR 2013i \\
\hline & Plum & Hexane:acetone & GC-ECD & & & Plum & Methanol & HPLC & \\
\hline \multirow[t]{3}{*}{ Fenpyroximate } & Grape & Acetonitrile:acetone & HPLC-MS/MS & JMPR $2010 \mathrm{~g}$ & & Tomato & Methanol & HPLC & \\
\hline & Orange & Acetonitrile:acetone & HPLC-MS/MS & & Trifloxystrobin & Strawberry & Acetonitrile:water & GC-MS/MS & JMPR 20121 \\
\hline & Tomato & Ethyl acetate & HPLC-MS/MS & & Triflumizole & Apple & Methanol:water & HPLC-UVD & JMPR 2013j \\
\hline \multirow[t]{3}{*}{ Flubendiamide } & Apple & Acetonitrile & HPLC-MS/MS & JMPR $2010 \mathrm{~h}$ & & Grape & Methanol & HPLC-UVD & \\
\hline & Grape & Acetonitrile & HPLC-MS/MS & & Triforine & Tomato & Acetone & HPLC-MS/MS & JMPR 20141 \\
\hline & Tomato & Acetonitrile & HPLC-MS/MS & & & & & & \\
\hline
\end{tabular}


Table 3 Processing factor of pesticides during apple processing

\begin{tabular}{|c|c|c|c|c|c|c|c|c|c|c|c|}
\hline \multirow{2}{*}{$\begin{array}{c}\text { Pesticide } \\
\text { (Application method) }\end{array}$} & \multirow{2}{*}{ Formulation } & \multirow{2}{*}{$\begin{array}{l}\text { Mode of } \\
\text { action }\end{array}$} & \multirow{2}{*}{$\log K_{o w}$} & \multirow{2}{*}{$\begin{array}{c}\text { Vapor pressure } \\
(\mathrm{mPa})\end{array}$} & \multicolumn{7}{|c|}{$\mathrm{PF}^{1)}$ by Processing method } \\
\hline & & & & & Washed & Dried & Canned & Juice & Sauce & Puree & $\begin{array}{l}\text { Pomace } \\
\text { (wet) }\end{array}$ \\
\hline Clothianidin (foliar) & $\mathrm{WG}^{2)}$ & $\mathrm{S}^{3)}$ & 0.893 & $3.8 \times 10^{-8}$ & & & & 0.14 & & & 0.24 \\
\hline Cyantraniliprole (foliar) & $\mathrm{SE}^{4)}$ & $\mathrm{N}^{5)}$ & 1.94 & $5.13 \times 10^{-15}$ & 0.58 & & 0.13 & 0.31 & 2.2 & 1.0 & 1.0 \\
\hline Cyflumetofen (foliar) & $\mathrm{SC}^{6)}$ & $\mathrm{N}$ & 4.3 & $5.9 \times 10^{-3}$ & & 0.5 & 0.1 & 0.23 & 2.7 & & 1.3 \\
\hline $\begin{array}{c}\text { Cyfluthrin } \\
\text { /beta-cyfluthrin } \\
\text { (foliar) }\end{array}$ & $\mathrm{WP}^{7)}$ & $\mathrm{N}$ & $\begin{array}{l}6.0(1) \\
5.9(2) \\
6.0(3) \\
5.9(4)\end{array}$ & $\begin{array}{l}9.6 \times 10^{-4}(1) \\
1.4 \times 10^{-5}(2) \\
2.1 \times 10^{-5}(3) \\
8.5 \times 10^{-4}(4)\end{array}$ & 0.67 & 0.2 & & N.D. & 0.23 & & \\
\hline Cyprodinil (foliar) & WG & $\mathrm{S}$ & 3.9 & 0.51 & & & & 0.03 & & & 3.5 \\
\hline Difenoconazole (foliar) & $\mathrm{EC}^{8)}$ & $\mathrm{S}$ & 4.4 & $3.3 \times 10^{-5}$ & & & & 0.03 & & & 9.5 \\
\hline Dithianon (foliar) & WG & $\mathrm{N}$ & 3.2 & $2.7 \times 10^{-6}$ & & 0.1 & N.D. & N.D. & N.D. & & 2.2 \\
\hline Emamectin benzoate (foliar) & $\mathrm{SG}^{9)}$ & $\mathrm{N}$ & 5.0 & $4 \times 10^{-3}$ & & & & N.D. & & & 5.1 \\
\hline Etofenprox (foliar) & $\mathrm{EC}$ & $\mathrm{N}$ & 6.9 & $8.134 \times 10^{-4}$ & 0.61 & & N.D. & N.D. & & 0.305 & 2.7 \\
\hline Flubendiamide (foliar) & $\mathrm{SC}$ & $\mathrm{N}$ & 4.2 & $<0.1$ & 0.97 & 0.51 & & 0.06 & 0.36 & & 7.5 \\
\hline Fluopyram (foliar) & $\mathrm{SC}$ & $\mathrm{N}$ & 3.3 & $1.2 \times 10^{-3}$ & 0.55 & 0.64 & & N.D. & 0.36 & & 2.3 \\
\hline Flutriafol (foliar) & $\mathrm{SC}$ & $\mathrm{S}$ & 2.3 & $7.1 \times 10^{-6}$ & & & & 0.5 & & & 1.9 \\
\hline Fluxapyroxad (foliar) & $\mathrm{EC}$ & $\mathrm{N}$ & 3.08 & $2.7 \times 10^{-6}$ & & 0.54 & 0.22 & 0.21 & 0.24 & & \\
\hline Myclobutanil (foliar) & WP & $\mathrm{S}$ & 2.94 & 0.198 & & & & 0.17 & & 0.3 & 1.61 \\
\hline Penthiopyrad (foliar) & $\mathrm{EC}$ & $\mathrm{N}$ & 4.228 & $6.43 \times 10^{-3}$ & & & N.D. & 0.07 & N.D. & & 5.1 \\
\hline Pyrimethanil (foliar) & $\mathrm{SC}$ & $\mathrm{T}^{10)}$ & 2.84 & 2.2 & & & & 0.45 & & 0.37 & 4.1 \\
\hline Sulfoxaflor (foliar) & $\mathrm{SC}$ & $\mathrm{N}$ & 3.99 & 14 & & & & 0.4 & 0.6 & & 1.1 \\
\hline Tebuconazole (foliar) & EC & $\mathrm{S}$ & 3.7 & $1.7 \times 10^{-3}$ & & 0.61 & & 0.23 & 0.34 & & 2.6 \\
\hline Triflumizole (foliar) & $\mathrm{WS}^{11)}$ & $\mathrm{S}$ & 5.06 & 0.186 & & & & 0.23 & 0.35 & & 0.9 \\
\hline Mean & & & & & $\begin{array}{c}0.68 \\
\pm 0.17\end{array}$ & $\begin{array}{c}0.44 \\
\pm 0.21\end{array}$ & $\begin{array}{c}0.08 \\
\pm 0.09\end{array}$ & $\begin{array}{c}0.16 \\
\pm 0.16\end{array}$ & $\begin{array}{c}0.67 \\
\pm 0.90\end{array}$ & $\begin{array}{c}0.49 \\
\pm 0.34\end{array}$ & $\begin{array}{c}3.10 \\
\pm 2.50\end{array}$ \\
\hline
\end{tabular}

${ }^{1)}$ Processing factor: Residues after processing/Residues of raw material ${ }^{2)}$ Water dispersible granule ${ }^{3)}$ Systemic ${ }^{4)}$ Suspo-emulsion ${ }^{5)}$ Non-systemic ${ }^{6}$ Suspension concentrate ${ }^{7)}$ Wettable powder ${ }^{8)}$ Emulsifiable concentrate ${ }^{9)}$ Water soluble granule ${ }^{10)}$ Translaminar ${ }^{11)}$ Water dispersible powder for slurry seed treatment

Table 4 Correlation between pesticide characteristics and processing methods of apple

\begin{tabular}{lc}
\hline \multicolumn{1}{c}{ Pesticide characteristics and processing methods } & $\begin{array}{c}\text { Correlation } \\
\text { coefficient (n) }\end{array}$ \\
\hline $\mathrm{K}_{\text {ow }}$ and juice (all samples) & $-0.468^{*}(19)$ \\
$\mathrm{K}_{\text {ow }}$ and juice (formulation of EC \& SC) & $-0.623 *(11)$ \\
$\mathrm{K}_{\mathrm{ow}}$ and juice (formulation of other) & $-0.467(8)$ \\
$\mathrm{K}_{\mathrm{ow}}$ and juice (mode of action: systemic) & $-0.299(8)$ \\
$\mathrm{K}_{\mathrm{ow}}$ and juice (mode of action: non systemic) & $-0.486(11)$ \\
\hline $\mathrm{K}_{\mathrm{ow}}$ and pomace (all samples) & $0.347(17)$ \\
$\mathrm{K}_{\mathrm{ow}}$ and pomace (formulation of EC \& SC) & $0.120(10)$ \\
$\mathrm{K}_{\mathrm{ow}}$ and pomace (formulation of other) & $0.637(7)$ \\
$\mathrm{K}_{\mathrm{ow}}$ and pomace (mode of action: systemic) & $0.427(8)$ \\
$\mathrm{K}_{\mathrm{ow}}$ and pomace (mode of action: non systemic) & $0.294(9)$ \\
\hline Vapor pressure and dried (all samples) & $0.158(7)$ \\
Vapor pressure and dried (formulation of EC \& SC) & $-0.476(5)$ \\
Vapor pressure and dried (formulation of other) & - \\
Vapor pressure and dried (mode of action: systemic) & - \\
Vapor pressure and dried (mode of action: non systemic) & $0.234(6)$ \\
\hline Vapor pressure and sauce (all samples) & $-0.028(11)$ \\
Vapor pressure and sauce (formulation of EC \& SC) & $-0.028(7)$ \\
Vapor pressure and sauce (formulation of other) & $-0.229(4)$ \\
Vapor pressure and sauce (mode of action: systemic) & - \\
Vapor pressure and sauce (mode of action: non systemic) & $-0.055(9)$ \\
\hline
\end{tabular}

*,**: Significant at 5 and $1 \%$ probability levels
조사되었다. 이들 성분들은 Table 5에서 농약의 특성을 살펴보 면 농약 살포 후 과육으로 침투하는 특성을 가진 농약으로 분 류된다. 또한 etoxazole을 제외한 3 가지 농약은 $\mathrm{K}_{\mathrm{ow}}$ 값이 수용 성에 가까운 성질이 반영되어 높은 PF 결과를 나타낸 것으로 판단되었다. 이들 4 가지 성분을 제외한 15 가지 농약 성분의 $\mathrm{PF}$ 는 상당량의 농약이 주스 제조 과정 중 제거되어 불검출-0.7로 조사되었다. 포도 주스의 $\mathrm{PF}$ 가 사과 주스 보다 높은 경향을 보 이나 두 작물에서 같은 농약들은 비슷한 결과를 보인다. 포도 주의 경우는 12 농약 성분의 $\mathrm{PF}$ 의 범위는 불검출-1.1(평균 0.36 \pm 0.41 ) 이었다. 이들 중 cyantraniliprole은 1, etoxazole 1.1, fenamidone 0.71 및 sulfoxaflor 0.7 인 경우를 제외한 나머지 8 성분은 불검출- 0.28 로 포도주 발효과정 중 농약 성분은 대부분 분해된다는 것을 알 수 있었다. 건포도는 17 개 농약 성분의 자 료 중 $\mathrm{PF}$ 의 범위는 0.22-6.29(평균 2.53 \pm 1.49 )이었다. Chlorothalonil 의 0.26 , cyantraniliprole 0.52 및 triflumizole 0.22 결과를 제 외한 14 개 농약의 $\mathrm{PF}$ 는 1.64-6.29로 건포도 제조과정 중 대부 분 농약성분은 농축됨을 알 수 있었다. 건포도의 전통적인 제 조 방법은 실온에서 장기간 건조하는 방법이며, 또 다른 방법 은 실온에서 건조 후 열풍으로 다시 건조하는 방식이다. 건포 도 제조 시 chlororthalonil 등 3가지 농약의 $\mathrm{PF}$ 값이 낮은 이 유는 열풍건조 방식으로 제조된 제품의 결과로 판단된다. 포도 에 대한 농약기준 설정 시 반드시 건포도에 대한 별도의 농약 
Table 5 Processing factor of pesticides during grape processing

\begin{tabular}{|c|c|c|c|c|c|c|c|c|c|}
\hline \multirow{2}{*}{$\begin{array}{c}\text { Pesticide } \\
\text { (Application method) }\end{array}$} & \multirow[b]{2}{*}{ Formulation } & \multirow{2}{*}{$\begin{array}{l}\text { Mode of } \\
\text { action }\end{array}$} & \multirow[b]{2}{*}{$\log K_{\text {ow }}$} & \multirow{2}{*}{$\begin{array}{l}\text { Vapor pressure } \\
\quad(\mathrm{mPa})\end{array}$} & \multicolumn{5}{|c|}{$\mathrm{PF}^{1)}$ by Processing method } \\
\hline & & & & & Washed & Juice & Wine & Raisins & $\begin{array}{l}\text { Pomace } \\
\text { (wet) }\end{array}$ \\
\hline Ametoctradin (foliar) & $\mathrm{SC}^{2)}$ & $\mathrm{N}^{3)}$ & 4.40 & $2.1 \times 10^{-7}$ & & & & 3.4 & 3.4 \\
\hline Chlorothalonil (foliar) & $\mathrm{SC}$ & $\mathrm{N}$ & 2.92 & $7.6 \times 10^{-2}$ & & 0.14 & N.D. & 0.26 & 1.3 \\
\hline Clothianidin (foliar) & $\mathrm{WG}^{4)}$ & $S^{5)}$ & 0.893 & $3.8 \times 10^{-8}$ & & 1.45 & & 2.6 & \\
\hline Cyantraniliprole (foliar) & $\mathrm{OD}^{6}$ & $\mathrm{~N}$ & 1.94 & $5.13 \times 10^{-15}$ & & 0.52 & 1.0 & 0.52 & 2.7 \\
\hline Cyflumetofen (foliar) & $\mathrm{SC}$ & $\mathrm{N}$ & 4.3 & $5.9 \times 10^{-3}$ & & 0.16 & 0.04 & & 2.2 \\
\hline Dichlobenil (foliar) & $\mathrm{SC}$ & S & 2.70 & 144 & & 1.4 & & 2.8 & \\
\hline Difenoconazole (foliar) & $\mathrm{EC}^{7)}$ & $\mathrm{S}$ & 4.4 & $3.3 \times 10^{-5}$ & & 0.46 & & 2.1 & \\
\hline Dinotefuran (foliar) & $\mathrm{SG}^{8)}$ & S & -0.549 & $<1.7 \times 10^{-3}$ & & 1.2 & & 3.7 & \\
\hline Dithianon (foliar) & $\begin{array}{l}\text { SC (wine) } \\
\text { WG (others) }\end{array}$ & $\mathrm{N}$ & 3.2 & $2.7 \times 10^{-6}$ & & N.D. & N.D. & 1.64 & 0.93 \\
\hline Etofenprox (foliar) & EC & $\mathrm{N}$ & 6.9 & $8.134 \times 10^{-4}$ & & N.D. & N.D. & 2.1 & \\
\hline Etoxazole (foliar) & WG & S & 5.59 & $7.0 \times 10^{-3}$ & & 1.7 & 1.1 & & \\
\hline Fenamidone (foliar) & WG & S & 2.8 & $3.4 \times 10^{-4}$ & & 0.36 & 0.71 & & 2.0 \\
\hline Fenpyroximate (foliar) & $\mathrm{SC}$ & $\mathrm{N}$ & 5.01 & $7.4 \times 10^{-3}$ & & N.D. & & 2.7 & 2.8 \\
\hline Flubendiamide (foliar) & $\mathrm{SC}$ & $\mathrm{N}$ & 4.2 & $<0.1$ & 0.45 & 0.13 & & 1.7 & 2.9 \\
\hline Fluopyram (foliar) & $\mathrm{SC}$ & $\mathrm{N}$ & 3.3 & $1.2 \times 10^{-3}$ & 0.62 & N.D. & 0.16 & 2.9 & 3.2 \\
\hline Flutriafol (foliar) & $\mathrm{SC}$ & S & 2.3 & $7.1 \times 10^{-6}$ & & 0.63 & & 2.8 & \\
\hline Metrafenone (foliar) & $\mathrm{SC}$ & $\mathrm{N}$ & 4.3 & $1.53 \times 10^{-1}$ & & 0.05 & 0.19 & 3.75 & 3.2 \\
\hline Myclobutanil (foliar) & $\mathrm{EW}^{9)}$ & S & 2.94 & 0.198 & & 0.2 & 0.14 & 6.29 & \\
\hline Sulfoxaflor (foliar) & $\mathrm{SC}$ & $\mathrm{N}$ & 3.99 & 14 & & 0.7 & 0.7 & 3.5 & 1.0 \\
\hline Tebuconazole (foliar) & WG & S & 3.7 & $1.7 \times 10^{-3}$ & & & 0.28 & & \\
\hline Triflumizole (foliar) & $\mathrm{WS}^{10)}$ & S & 5.06 & 0.186 & & 0.42 & & 0.22 & 4.32 \\
\hline $\mathrm{Me}$ & & & & & $0.53 \pm 0.12$ & $050 \pm 0.55$ & $0.36 \pm 0.41$ & $2.53 \pm 1.49$ & $2.50 \pm 1.04$ \\
\hline
\end{tabular}

${ }^{1}$ Processing factor: Residues after processing/Residues of raw material ${ }^{2}$ Suspension concentrate ${ }^{3}$ Non-systemic ${ }^{4}$ Water dispersible granule ${ }^{5}$ Systemic ${ }^{6}$ Oil dispersion ${ }^{7)}$ Emulsifiable concentrate ${ }^{8}$ Water soluble granule ${ }^{9)}$ Emulsion oil in water ${ }^{10}$ Water dispersible powder for slurry seed treatment

Table 6 Correlation between pesticide characteristics and processing methods of grape

\begin{tabular}{lc}
\hline \multicolumn{1}{c}{ Pesticide characteristics and processing methods } & $\begin{array}{c}\text { Correlation } \\
\text { coefficient (n) }\end{array}$ \\
\hline $\mathrm{K}_{\text {ow }}$ and juice (all samples) & $-0.380(19)$ \\
$\mathrm{K}_{\text {ow }}$ and juice (formulation of EC \& SC) & $-0.455(12)$ \\
$\mathrm{K}_{\text {ow }}$ and juice (formulation of other) & $-0.094(7)$ \\
$\mathrm{K}_{\text {ow }}$ and juice (mode of action: systemic) & $-0.652(9)$ \\
$\mathrm{K}_{\text {ow }}$ and juice (mode of action: non systemic) & $0.180(10)$ \\
\hline $\mathrm{K}_{\text {ow }}$ and wine (all samples) & $-0.114(12)$ \\
$\mathrm{K}_{\text {ow }}$ and wine (formulation of EC \& SC) & $-0.094(7)$ \\
$\mathrm{K}_{\text {ow }}$ and wine (formulation of other) & $0.239(5)$ \\
$\mathrm{K}_{\text {ow }}$ and wine (mode of action: systemic) & $-0.418(4)$ \\
$\mathrm{K}_{\text {ow }}$ and wine (mode of action: non systemic) & $-0.096(8)$ \\
\hline $\mathrm{K}_{\text {ow }}$ and pomace (all samples) & $0.464(12)$ \\
$\mathrm{K}_{\text {ow }}$ and pomace (formulation of EC \& SC) & $0.530(9)$ \\
$\mathrm{K}_{\text {ow }}$ and pomace (formulation of other) & $0.843(3)$ \\
$\mathrm{K}_{\text {ow }}$ and pomace (mode of action: systemic) & - \\
$\mathrm{K}_{\text {ow }}$ and pomace (mode of action: non systemic) & $0.284(10)$ \\
\hline Vapor pressure and raisin (all samples) & $0.064(17)$ \\
Vapor pressure and raisin (formulation of EC \& SC) & $0.139(12)$ \\
Vapor pressure and raisin (formulation of other) & $0.253(5)$ \\
Vapor pressure and raisin (mode of action: systemic) & $-0.031(7)$ \\
Vapor pressure and raisin (mode of action: non systemic) & $0.362(10)$ \\
\hline$* * *$ Significant at 5 and $1 \%$ probability levels & \\
\hline
\end{tabular}

가공계수 실험결과가 제출되어 평가되어야 할 것으로 판단되었 다. Cabras 등(1998b)은 건포도 제조 후 benalaxyl, dimethoate, iprodione, metalaxyl, phosalone, procymidone 및 vinclozolin의 잔류량을 비교한 연구에서 14 개 처리구 중 3 개 구에서만 가공 계수가 1 이상의 결과를 보였으며, Lentza-Rizos 등(2006)은 건 포도 제조 중 azoxystrobin 잔류량을 품종 별로 연구한 결과에 는 5 개 처리구 중 3 개 구에서만 가공계수가 1 이상의 결과를 나타냈다. Kaushik 등(2009)의 연구결과에는 건포도 제조 후 dimethoate 및 methamidofos의 농약 잔류량이 원료 잔류량보다 감소된다고 보고하였다. 이와 같이 건포도 제조 시 농약 잔류 량 변화에 대한 일반 연구논문과 $\mathrm{CODEX}$ 기준 설정을 위한 연 구 결과에 다소 차이가 있는 것으로 조사되었다. $\mathrm{CODEX}$ 에서 는 농약 기준 설정시 극단적인 상황을 감안하여 기준을 설정하 기 때문에 건포도 가공 시 가능한 한 단순하면서 최대한 높은 농도로 잔류되는 가공처리를 하도록 권고하고 있어서 농약의 $\mathrm{PF}$ 값이 일반 연구 논문들의 결과보다 높은 경향으로 나타난 것으로 판단된다.

Table 6에서 포도 가공 시 농약의 물리화학적 특성과의 상관 관계를 조사한 결과, $\mathrm{K}_{\mathrm{ow}}$ 와 포도 주스, pomace 및 포도주 제조 후 농약의 $\mathrm{PF}$ 는 무의미한 낮은 상관 관계를 보였으며, 증기압 과 건포도 제조에서도 무의미한 낮은 상관 관계가 조사되었다. 포도의 가공 공정에는 농약의 특성보다는 가공방법에 따라서 농 약의 $\mathrm{PF}$ 값이 결정될 수 있다는 것으로 판단된다. 
Table 7 Processing factor of pesticides during orange processing

\begin{tabular}{|c|c|c|c|c|c|c|c|c|c|c|}
\hline \multirow{2}{*}{$\begin{array}{c}\text { Pesticide } \\
\text { (Application method) }\end{array}$} & \multirow[b]{2}{*}{ Formulation } & \multirow{2}{*}{$\begin{array}{l}\text { Mode of } \\
\text { action }\end{array}$} & \multirow[b]{2}{*}{$\log K_{\text {ow }}$} & \multirow{2}{*}{$\begin{array}{l}\text { Vapor pressure } \\
(\mathrm{mPa})\end{array}$} & \multicolumn{6}{|c|}{$\mathrm{PF}^{1)}$ by Processing method } \\
\hline & & & & & Peel & Juice & Oil & Marmalade & Molasses & $\begin{array}{l}\text { Pomace } \\
\text { (wet) }\end{array}$ \\
\hline Boscalid (foliar) & $\mathrm{WG}^{2)}$ & $\mathrm{T}^{3)}$ & 2.96 & $7.2 \times 10^{-4}$ & & N.D. & 59 & & & \\
\hline Chlorantraniliprole (foliar) & $\mathrm{SC}^{4)}$ & $\mathrm{N}^{5)}$ & 2.77 & $6.3 \times 10^{-12}$ & & 0.17 & & & & \\
\hline Chlorfenapyr & $\mathrm{EC}^{6)}$ & $S^{7)}$ & 4.83 & $<1.2 \times 10^{-2}$ & 0.78 & 0.015 & 70 & & & \\
\hline Cyantraniliprole (foliar) & $\mathrm{OD}^{8)}$ & $\mathrm{N}$ & 1.94 & $5.13 \times 10^{-15}$ & & N.D. & 8.5 & N.D. & 0.59 & \\
\hline Cyflumetofen (foliar) & $\mathrm{SC}$ & $\mathrm{N}$ & 4.3 & $5.9 \times 10^{-3}$ & 2.92 & 0.05 & 120 & 0.026 & N.D. & \\
\hline $\begin{array}{l}\text { Cyfluthrin } \\
\text { /beta-cyfluthrin } \\
\text { (foliar) }\end{array}$ & $\mathrm{EC}$ & $\mathrm{N}$ & $\begin{array}{l}6.0(1) \\
5.9(2) \\
6.0(3) \\
5.9(4)\end{array}$ & $\begin{array}{l}9.6 \times 10^{-4},(1) \\
1.4 \times 10^{-5},(2) \\
2.1 \times 10^{-5},(3) \\
8.5 \times 10^{-4},(4)\end{array}$ & 1.2 & N.D. & 0.01 & & 16 & \\
\hline Difenoconazole (foliar) & $\mathrm{EC}$ & $\mathrm{S}$ & 4.4 & $3.3 \times 10^{-5}$ & & N.D. & 47 & & & \\
\hline Etoxazole (foliar) & $\mathrm{SC}$ & $\mathrm{S}$ & 5.59 & $7.0 \times 10^{-3}$ & & 0.5 & & & & 1.5 \\
\hline Fenbuconazole (foliar) & $\mathrm{SC}$ & $\mathrm{P}^{9)}$ & 3.23 & $3.4 \times 10^{-1}$ & & N.D. & 58 & & & \\
\hline Fenpropathrin (foliar) & $\mathrm{EC}$ & $\mathrm{N}$ & 6 & 0.730 & 2.82 & N.D. & 50.1 & & & \\
\hline Fenpropathrin (foliar) & $\mathrm{EC}$ & $\mathrm{N}$ & 6 & 0.730 & & 0.12 & & & & 9.8 \\
\hline Fenpyroximate (foliar) & $\mathrm{EC}$ & $\mathrm{N}$ & 5.01 & $7.4 \times 10^{-3}$ & & 0.13 & & & & \\
\hline Fludioxonil (foliar) & $\mathrm{SC}$ & $\mathrm{N}$ & 4.12 & $3.9 \times 10^{-4}$ & & 0.11 & & & & \\
\hline Fluopyram (foliar) & $\mathrm{SC}$ & $\mathrm{N}$ & 3.3 & $1.2 \times 10^{-3}$ & 1.8 & 0.01 & 16 & & & \\
\hline $\begin{array}{l}\text { Glufosinate ammonium } \\
\text { (direct spray) }\end{array}$ & $\mathrm{SL}^{10)}$ & $\mathrm{S}$ & $<0.1$ & $<0.1$ & & 0.71 & N.D. & & 2.62 & \\
\hline Methoxyfenozide (foliar) & $\mathrm{SC}$ & $\mathrm{N}$ & 3.7 & $<1.48 \times 10^{-3}$ & & 0.22 & 42.5 & 0.77 & & \\
\hline $\begin{array}{c}\text { Propiconazole } \\
\text { (dip/drench or coating) }\end{array}$ & $\mathrm{WP}^{11)}$ & $\mathrm{S}$ & 3.72 & $2.7 \times 10^{-2}$ & & N.D & 18.5 & & & \\
\hline Pyraclostrobin (foliar) & $\mathrm{EC}$ & $\mathrm{T}$ & 3.99 & $2.6 \times 10^{-5}$ & 4.6 & 0.04 & 6.24 & 0.18 & & 1.41 \\
\hline Sulfoxaflor (foliar) & $\mathrm{SC}$ & $\mathrm{N}$ & 3.99 & 14 & 5.6 & 0.14 & N.D. & & & \\
\hline Tolfenpyrad (foliar) & $\mathrm{EC}$ & $\mathrm{N}$ & 5.61 & $5 \times 10^{-3}$ & & & & & & \\
\hline
\end{tabular}

${ }^{1)}$ Processing factor: Residues after processing/Residues of raw material ${ }^{2)}$ Water dispersible granule ${ }^{3)}$ Translaminar ${ }^{4}$ Suspension concentrate ${ }^{5)}$ Non-systemic ${ }^{6}$ Emulsifiable concentrate ${ }^{7}$ Systemic ${ }^{8}$ Oil dispersion ${ }^{9}$ Penetrant ${ }^{10}$ Soluble concentrate ${ }^{11)}$ Wettable powder

\section{오렌지의 잔류농약 가공계수}

Table 7은 오렌지에 대한 19종 농약의 PF 연구자료 중 껍질, 주스, 오일, 마멀레이드, molasses 및 pomace의 가공방법 별로 정리 - 비교하였다. 오렌지의 대표적인 가공품인 주스는 불검출 -0.71 (평균 $0.12 \pm 0.19$ )로 18 종 농약의 $\mathrm{PF}$ 값이 1 미만을 나타내 었다. 오렌지 주스는 원료를 세척, 분쇄하고 여과 또는 압착한 후 살균하는 비교적 단순한 가공공정으로 제조된다. 오렌지 주 스 가공 후 boscalid, cyantraniliprole, cyfluthrin, difenoconazole, fenbuconazole, fenpropathrin 및 propiconazole 농약은 불검출이 었으며, chlorfenapyr, cyflumetofen, fluopyram 및 pyraclostrobin 농약의 $\mathrm{PF}$ 값은 각각 $0.015,0.05,0.01$ 및 0.04 로 매우 낮은 수준을 나타내었다. 수용성 특성을 가진 glufosinate ammonium 의 PF가 0.71 인 경우를 제외하면 오렌지 주스 가공 후에는 잔 류농약이 거의 이행되지 않는 것으로 조사되었다. 다만 침투성 농약인 etoxazole 농약의 경우 PF가 0.5 로 조사되었다. 오렌지 오일은 원료를 세척, 분쇄 및 원심 분리하여 주스와 오일로 분 리하여 제조된다. 오일의 경우에는 수용성 농약인 glufosinate ammonium, 비침투성 농약인 sulfoxaflor 및 cyfluthrin의 PF가 각각 불검출, 불검출 및 0.01 로 조사되었으나, 나머지 16 종 농 약의 $\mathrm{PF}$ 는 6.24-120으로 높게 나타나서 이들 농약의 지용성이 반영된 것을 알 수 있었다. Glufosinate ammonium은 수용성
Table 8 Correlation between pesticide characteristics and processing methods of orange

Pesticide characteristics and processing methods Correlation coefficient (n)

\begin{tabular}{lc}
\hline $\mathrm{K}_{\text {ow }}$ and juice (all samples) & $-0.364(19)$ \\
$\mathrm{K}_{\text {ow }}$ and juice (formulation of EC \& SC) & $0.101(15)$ \\
$\mathrm{K}_{\text {ow }}$ and juice (formulation of other) & - \\
$\mathrm{K}_{\text {ow }}$ and juice (mode of action: systemic) & $-0.902 * *(8)$ \\
$\mathrm{K}_{\text {ow }}$ and juice (mode of action: non systemic) & $0.163(11)$ \\
\hline $\mathrm{K}_{\text {ow }}$ and oil (all samples) & $0.270(14)$ \\
$\mathrm{K}_{\text {ow }}$ and oil (formulation of EC \& SC) & $-0.027(10)$ \\
$\mathrm{K}_{\text {ow }}$ and oil (formulation of other) & $0.590(4)$ \\
$\mathrm{K}_{\text {ow }}$ and oil (mode of action: systemic) & $0.539(7)$ \\
$\mathrm{K}_{\text {ow }}$ and oil (mode of action: non systemic) & $0.142(7)$ \\
\hline
\end{tabular}

*,**: Significant at 5 and $1 \%$ probability levels

특성을 가지고 있으므로 오일에서 불검출 결과를 나타냈지만, 지용성에 가까운 $\mathrm{K}_{\mathrm{ow}}$ 를 가지는 sulfoxaflor 및 cyfluthrin의 경우 에는 농약의 특성과 전혀 다른 결과를 보였다. 오렌지가 오일 로 제조되어 식용으로 사용될 경우에는 농약잔류허용기준 설정 
Table 9 Processing factor of pesticides during tomato processing

\begin{tabular}{|c|c|c|c|c|c|c|c|c|c|c|c|c|}
\hline \multirow{2}{*}{$\begin{array}{c}\text { Pesticide } \\
\text { (Application method) }\end{array}$} & \multirow{2}{*}{ Formulation } & \multirow{2}{*}{$\begin{array}{l}\text { Mode of } \\
\text { action }\end{array}$} & \multirow[b]{2}{*}{$\log \mathrm{K}_{\mathrm{ow}}$} & \multirow{2}{*}{$\begin{array}{l}\text { Vapor pressure } \\
(\mathrm{mPa})\end{array}$} & \multicolumn{8}{|c|}{$\mathrm{PF}^{1)}$ by Processing method } \\
\hline & & & & & Washed & Peeled & Canned & Juice & Puree & Paste & Ketchup & $\begin{array}{c}\text { Pomace } \\
\text { (wet) }\end{array}$ \\
\hline Ametoctradin (foliar) & $\mathrm{SC}^{2)}$ & $\mathrm{N}^{3)}$ & 4.40 & $2.1 \times 10^{-7}$ & & & & & & & & 1.3 \\
\hline Bifenthrin (foliar) & $\mathrm{EC}^{4)}$ & $\mathrm{N}$ & $>6$ & $2.4 \times 10^{-2}$ & & & & & N.D. & N.D. & & \\
\hline Chlorfenapyr (foliar) & $\mathrm{SC}$ & $\mathrm{S}^{5)}$ & 4.83 & $<1.2 \times 10^{-2}$ & 0.78 & & & 0.28 & 0.94 & 1.8 & 0.83 & 63 \\
\hline Clothianidin (foliar) & $\mathrm{WG}^{6)}$ & $\mathrm{S}$ & 0.893 & $3.8 \times 10^{-8}$ & & & & & 0.77 & 5.9 & & \\
\hline Cyantraniliprole (foliar) & $\mathrm{OD}^{7)}$ & $\mathrm{N}$ & 1.94 & $5.13 \times 10^{-15}$ & 0.17 & N.D. & N.D. & N.D. & 0.25 & 0.86 & & \\
\hline Cycloxydim (foliar) & $\mathrm{EC}$ & $A^{8)}$ & 1.36 & 0.01 & 1.25 & 1.25 & 0.57 & 1.11 & 3.71 & & 1.57 & 1.38 \\
\hline Cyflumetofen (foliar) & $\mathrm{SC}$ & $\mathrm{N}$ & 4.3 & $5.9 \times 10^{-3}$ & & & 0.2 & 0.2 & 0.59 & 0.3 & & 3.4 \\
\hline $\begin{array}{l}\text { Cyfluthrin } \\
\text { /beta-cyfluthrin } \\
\text { (foliar) }\end{array}$ & $\mathrm{EC}$ & $\mathrm{N}$ & $\begin{array}{l}6.0(1) \\
5.9(2) \\
6.0(3) \\
5.9(4)\end{array}$ & $\begin{array}{l}9.6 \times 10^{-4},(1) \\
1.4 \times 10^{-5},(2) \\
2.1 \times 10^{-5},(3) \\
8.5 \times 10^{-4},(4)\end{array}$ & & & & 0.3 & 0.7 & 1.8 & 0.8 & 6.5 \\
\hline Cyprodinil (foliar) & WG & $\mathrm{S}$ & 3.9 & 0.51 & & & & 0.17 & 0.52 & 1.565 & & \\
\hline Difenoconazole (foliar) & $\mathrm{EC}$ & $\mathrm{S}$ & 4.4 & $3.3 \times 10^{-5}$ & & & & & 0.57 & 1.6 & & \\
\hline Dinotefuran (foliar) & $\mathrm{SG}^{9)}$ & $\mathrm{S}$ & -0.549 & $<1.7 \times 10^{-3}$ & & & & & 1.6 & 4.6 & & \\
\hline Fenamidone (foliar) & WG & $\mathrm{S}$ & 2.8 & $3.4 \times 10^{-4}$ & & & 0.45 & 0.8 & 2.1 & 3.65 & 2.4 & 4.5 \\
\hline Fenpyroximate (foliar) & $\mathrm{EC}$ & $\mathrm{N}$ & 5.01 & $7.4 \times 10^{-3}$ & & & & & 0.41 & 0.56 & & \\
\hline Flubendiamide (foliar) & $\mathrm{SC}$ & $\mathrm{N}$ & 4.2 & $<0.1$ & 0.61 & 0.3 & 0.24 & 0.49 & 1.8 & 4.0 & & 3.13 \\
\hline Fludioxonil (drip/drench) & & $\mathrm{N}$ & 4.12 & $3.9 \times 10^{-4}$ & & & & & 0.36 & 0.33 & & \\
\hline Fluensulfone (spray) & & $\mathrm{N}$ & 5.0 & $7.4 \times 10^{-3}$ & & 0.33 & 0.33 & 0.75 & 1 & 1.8 & & 2.6 \\
\hline Fluopyram (foliar) & $\mathrm{SC}$ & $\mathrm{N}$ & 3.3 & $1.2 \times 10^{-3}$ & 0.67 & & & 0.36 & 0.73 & 0.46 & & 0.1 \\
\hline Fluxapyroxad (foliar) & $\mathrm{EC}$ & $\mathrm{N}$ & 3.08 & $2.7 \times 10^{-6}$ & 0.74 & 0.08 & 0.19 & 0.18 & 0.37 & 0.73 & & 3.4 \\
\hline Metrafenone (foliar) & $\mathrm{SC}$ & $\mathrm{N}$ & 4.3 & $1.53 \times 10^{-1}$ & & & & 0.34 & 0.81 & 0.385 & & 5.5 \\
\hline Myclobutanil (foliar) & $\mathrm{WP}^{10)}$ & $\mathrm{S}$ & 2.94 & 0.198 & & & & 0.5 & 1.33 & 3.92 & & \\
\hline Novaluron (foliar) & $\mathrm{EC}$ & $\mathrm{N}$ & 4.3 & $1.6 \times 10^{-2}$ & & & & & N.D. & 1.1 & & \\
\hline Penthiopyrad (foliar) & $\mathrm{SC}$ & $\mathrm{N}$ & 4.228 & $6.43 \times 10^{-3}$ & & & 0.07 & 0.27 & 2.1 & 3.6 & 1.1 & 5.1 \\
\hline Propamocarb (foliar) & $\mathrm{SC}$ & $\mathrm{S}$ & 0.84 & 730 & & & & 0.53 & 0.77 & 3.1 & & \\
\hline Sulfoxaflor (foliar) & $\mathrm{SC}$ & $\mathrm{N}$ & 3.99 & 14 & & & & 1 & 2 & 4.4 & 2.1 & \\
\hline Tebuconazole (foliar) & $\mathrm{SC}$ & $\mathrm{S}$ & 3.7 & $1.7 \times 10^{-3}$ & & & & 0.55 & 0.33 & 3.2 & & \\
\hline Tolfenpyrad (foliar) & $\mathrm{EC}$ & $\mathrm{N}$ & 5.61 & $5 \times 10^{-3}$ & & & & & 0.31 & 1.0 & & \\
\hline Triforine (foliar) & $\mathrm{EC}$ & $\mathrm{S}$ & 2.2 & 0.8 & & & & 0.75 & 2.3 & N.D. & & N.D. \\
\hline Mean & & & & & $\begin{array}{c}0.70 \\
\pm 0.35\end{array}$ & $\begin{array}{l}0.39 \\
\pm .50\end{array}$ & $\begin{array}{c}0.26 \\
\pm 0.19\end{array}$ & $\begin{array}{c}0.48 \\
\pm 0.30\end{array}$ & $\begin{array}{c}1.01 \\
\pm 0.87\end{array}$ & $\begin{array}{c}2.03 \\
\pm 1.70\end{array}$ & $\begin{array}{c}1.47 \\
\pm 0.67\end{array}$ & $\begin{array}{c}7.69 \\
\pm 16.74\end{array}$ \\
\hline
\end{tabular}

${ }^{1)}$ Processing factor: Residues after processing/Residues of raw material ${ }^{2)}$ Suspension concentrate ${ }^{3)}$ Non-systemic ${ }^{4)}$ Emulsifiable concentrate ${ }^{5)}$ Systemic ${ }^{6)}$ Water dispersible granule ${ }^{7)}$ Oil dispersion ${ }^{8}$ Absorbed ${ }^{9}$ Water soluble granule ${ }^{10)}$ Wettable powder

이 필요할 것으로 판단되었다.

Table 8에 오렌지 주스 및 오일 제조 후 농약의 $\mathrm{PF}$ 값과 $\mathrm{K}_{\mathrm{ow}}$ 의 상관 관계를 분석하였다. 오렌지 주스 전체 시료와 $\mathrm{K}_{\mathrm{ow}}$ 는 무의미한 낮은 상관관계가 나타났으나, 침투성 농약으로 처리된 오렌지를 주스로 제조한 경우는 $\mathrm{K}_{\mathrm{ow}}$ 와 고도로 유의한 부상관 관계가 나타났다. 이 결과는 오렌지에 살포된 농약이 살포된 후 껍질과 과육으로 침투되었으나, 지용성이 강한 성분일수록 주스 로 이행되지 않음을 알 수 있었다. 살포된 농약 제형에 따른 주 스의 $\mathrm{PF}$ 와 $\mathrm{K}_{\mathrm{ow}}$ 는 무상관 관계를 보였으며, 오렌지 오일의 경우 에도 농약의 물리화학적 특성 및 농약의 $\mathrm{PF}$ 간에 무의미한 낮 은 상관관계가 나타났다.

\section{토마토의 잔류농약 가공계수}

Table 9에서는 토마토에 대한 27종 농약의 PF 자료 중 세척, 껍질 벗기기, 통조림, 주스, 퓌레, 페이스트, 케첩 및 pomace로
가공방법 별로 정리하여 비교하였다. 토마토 통조림은 세척, 박 피, 캔에 담기 및 살균 공정을 거쳐 제조되는데, 8 개 농약의 $\mathrm{PF}$ 는 불검출- 0.57 (평균 $0.26 \pm 0.19$ )로 나타났다. 사과 통조림에 비 하여 평균이 높은 $\mathrm{PF}$ 값을 보였지만 같은 농약에서는 비슷한 양상을 보였다. 주스는 세척, 분쇄, 데치기, 여과, 살균 과정을 거치게 되는데, $\mathrm{PF}$ 값은 불검출-1.11(평균 $0.48 \pm 0.30$ )로 조사되 었다. 처리된 27종 농약 중 cycloxydim 및 sulfoxaflor을 제외 하면, 불검출- 0.8 의 $\mathrm{PF}$ 값을 보여 사과와 오렌지 주스의 $\mathrm{PF}$ 값 보다 높게 조사되었다. 그러나 포도 주스의 $\mathrm{PF}$ 값과 유사한 경 향을 보였다. 토마토 주스 가공 시 껍질의 분리는 세척, 분쇄, 데 치기 후에 이루어지므로 잔류농약이 주스로 이행될 확률이 상 승될 수 있다. Cycloxydim은 흡수 이행되는 농약으로 PF 값이 1.11 로 가장 높게 나타났으나, sulfoxaflor의 경우는 비침투성임 에도 불구하고 1 의 값으로 조사되어서 향후 이들 농약 성분들 에 대한 가공 연구가 필요할 것으로 판단된다. 퓌레의 $\mathrm{PF}$ 는 불 
Table 10 Correlation between pesticide characteristics and processing methods of tomato

\begin{tabular}{|c|c|}
\hline Pesticide characteristics and processing methods & $\begin{array}{c}\text { Correlation } \\
\text { coefficient }(n)\end{array}$ \\
\hline $\begin{array}{l}\mathrm{K}_{\mathrm{ow}} \text { and juice (all samples) } \\
\mathrm{K}_{\mathrm{ow}} \text { and juice (formulation of EC \& SC) } \\
\mathrm{K}_{\mathrm{ow}} \text { and juice (formulation of other) } \\
\mathrm{K}_{\mathrm{ow}} \text { and juice (mode of action: systemic) } \\
\mathrm{K}_{\mathrm{ow}} \text { and juice (mode of action: non systemic) }\end{array}$ & $\begin{array}{c}-0.266(18) \\
-0.419(13) \\
0.137(5) \\
-0.669(8) \\
0.385(10)\end{array}$ \\
\hline $\begin{array}{l}\mathrm{K}_{\mathrm{ow}} \text { and puree (all samples) } \\
\mathrm{K}_{\mathrm{ow}} \text { and puree (formulation of EC \& SC) } \\
\mathrm{K}_{\mathrm{ow}} \text { and puree (formulation of other) } \\
\mathrm{K}_{\mathrm{ow}} \text { and puree (mode of action: systemic) } \\
\mathrm{K}_{\mathrm{ow}} \text { and puree (mode of action: non systemic) }\end{array}$ & $\begin{array}{l}-0.401(26) \\
-0.526^{*}(18) \\
-0.275(8) \\
-0.375(11) \\
-0.083(15)\end{array}$ \\
\hline $\begin{array}{l}\mathrm{K}_{\mathrm{ow}} \text { and paste (all samples) } \\
\mathrm{K}_{\mathrm{ow}} \text { and paste (formulation of EC \& SC) } \\
\mathrm{K}_{\mathrm{ow}} \text { and paste (formulation of other) } \\
\mathrm{K}_{\mathrm{ow}} \text { and paste (mode of action: systemic) } \\
\mathrm{K}_{\mathrm{ow}} \text { and paste (mode of action: non systemic) }\end{array}$ & $\begin{array}{l}-0.486(25) \\
-0.180(17) \\
-0.673(8) \\
-0.524(11) \\
-0.031(15)\end{array}$ \\
\hline $\begin{array}{l}\text { Vapor pressure and puree (all samples) } \\
\text { Vapor pressure and puree (formulation of EC \& SC) } \\
\text { Vapor pressure and puree (formulation of other) } \\
\text { Vapor pressure and puree (mode of action: systemic) } \\
\text { Vapor pressure and puree(mode of action: non systemic) }\end{array}$ & $\begin{array}{l}-0.052(26) \\
-0.050(18) \\
-0.337(8) \\
-0.227(11) \\
0.506(15)\end{array}$ \\
\hline $\begin{array}{l}\text { Vapor pressure and paste (all samples) } \\
\text { Vapor pressure and paste (formulation of EC \& SC) } \\
\text { Vapor pressure and paste (formulation of other) } \\
\text { Vapor pressure and paste(mode of action: systemic) } \\
\text { Vapor pressure and paste(mode of action: non systemic) }\end{array}$ & $\begin{array}{l}0.137(25) \\
0.264(19) \\
-0.171(6) \\
0.003(10) \\
0.576^{*}(15)\end{array}$ \\
\hline
\end{tabular}

*,**: Significant at 5 and $1 \%$ probability levels

검출-3.71(평균 $1.01 \pm 0.87$ ), 페이스트는 불검출-5.9(평균 $2.03 \pm 1.70$ ) 로 조사되었다. 퓌레는 토마토 주스를 $12-12.5^{\circ} \mathrm{Brix}$ 로 농축한 후 살균하여 제조하고, 페이스트는 주스를 $20-30^{\circ} \mathrm{Brix}$ 로 농축하여 수분을 제거한 다음 살균하여 제조한다. 이런 가공 특성으로 인
하여 퓌레보다 페이스트에서 PF 값이 높은 것으로 조사되었다 고 판단된다. 토마토 케첩의 경우에는 주스를 농축한 후 당, 식 초, 소금이 첨가된 후 살균 과정을 거쳐서 제조된다. 가공 후 $\mathrm{PF}$ 가 0.8-2.4(평균 $1.47 \pm 0.67$ )로 퓌레와 페이스트의 중간 정도 의 경향을 보였다. 토마토 가공품의 경우 원료 토마토에 대한 농약기준 설정 시 퓌레, 페이스트, 케첩에 대한 가공계수 연구 자료가 제출되어서 검토되어야 할 것으로 판단된다.

Table 10 에 토마토 가공품의 농약 $\mathrm{PF}$ 와 농약의 특성간 상관 관계를 비교한 결과를 나타내었다. $\mathrm{K}_{\mathrm{ow}}$ 와 퓌레의 $\mathrm{EC}$ 및 $\mathrm{SC}$ 제 형 $\mathrm{PF}$ 값 간에는 유의한 부상관 관계가 있었고, 비침투성 농약 이 살포된 토마토로 제조된 페이스트의 $\mathrm{PF}$ 와 증기압 사이도 유 의한 정상관관계가 있었다. 그 외는 $\mathrm{K}_{\mathrm{ow}}$ 및 증기압과 토마토 주스, 페이스트 및 퓌레의 $\mathrm{PF}$ 간에는 무의미한 낮은 상관 관계 가 조사되었다. 토마토 가공 시 농약의 특징과 $\mathrm{PF}$ 간의 상관관 계를 성립시키기 위한 면밀한 연구가 필요할 것으로 판단되었다.

\section{자두, 딸기 및 기타의 잔류농약 가공계수}

Table 11 에서는 자두에 대한 12 종 농약의 가공계수 자료에 대 하여 정리 - 비교하였다. 자두의 경우는 건조에 대한 가공계수 는 0.515-4.59(평균 2.12 \pm 1.11 )로 dithianon의 0.515 를 제외한 11 종 성분 전체가 1 이상의 가공계수를 나타냈다. 자두 건조 후 수분 증발로 인하여 농약 성분이 농축됨을 알 수 있었다. 자두 건조 후 건자두의 $\mathrm{PF}$ 와 증기압 간의 상관 관계는 무의미한 것 으로 조사되었지만(Table 12), 원료 자두 농약기준 설정 시 자 두의 건조 가공계수 연구자료의 검토가 필요할 것으로 사료된 다. 체리 및 망고의 건조 가공 $\mathrm{PF}$ 는 각각 5.1 및 6.3이였다.

Table 13의 딸기에 대한 자료 중 쨈의 경우에는 PF가 0.210.665 (평균 $0.49 \pm 0.18$ )로 조사되었다. 쨈 제조 시에는 수분 증발 을 위하여 장시간 동안의 가열과정이 있고 또한 농축 과정도 있다. 농약의 농축에도 불구하고 열에 의한 농약의 분해로 인 하여 $51 \%$ 정도가 감소 되었다고 판단하였다. 딸기 쨈 중 기타

Table 11 Processing factor of pesticides during plum processing

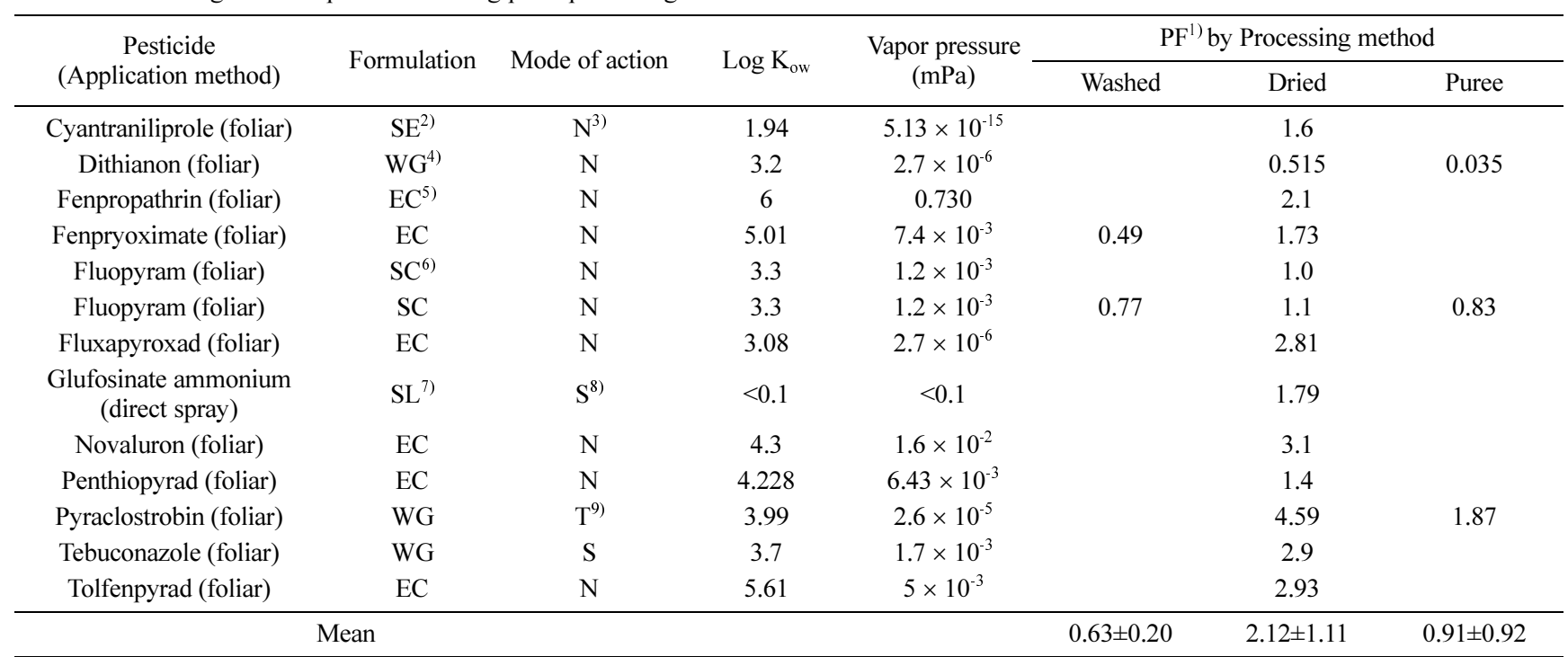

${ }^{1)}$ Processing factor: Residues after processing/Residues of raw material ${ }^{2)}$ Suspo-emulsion ${ }^{3)}$ Non-systemic ${ }^{4)}$ Water dispersible granule ${ }^{5)}$ Emulsifiable concentrate ${ }^{6)}$ Suspension concentrate ${ }^{7)}$ Soluble concentrate ${ }^{8)}$ Systemic ${ }^{9}$ Translaminar 
Table 12 Correlation between pesticide characteristics and processing methods of plum

\begin{tabular}{lc}
\hline \multicolumn{1}{c}{ Pesticide characteristics and processing methods } & $\begin{array}{c}\text { Correlation } \\
\text { coefficient (n) }\end{array}$ \\
\hline Vapor pressure and dried (all samples) & $-0.014(13)$ \\
Vapor pressure and dried (formulation of EC \& SC) & $0.047(8)$ \\
Vapor pressure and dried (formulation of other) & $-0.174(5)$ \\
Vapor pressure and dried (mode of action: systemic) & $-0.809(3)$ \\
Vapor pressure and dried (mode of action: non systemic) & $0.120(10)$ \\
\hline
\end{tabular}

*,**: Significant at 5 and $1 \%$ probability levels

제형 및 비침투성으로 분류된 $\mathrm{PF}$ 와 증기압 사이에 각각 정 및 부로 유의한 상관관계가 있었다(Table 14).

\section{초 록}

과일류 가공 중 농약의 가공계수는 과일 가공품의 농약 기준 설정에 사용된다. 본 연구는 2010년부터 2014년까지 JMPR의 가공식품 중 잔류농약 가공계수 자료를 고찰하였다. 사과의 세 척, 건조, 통조림, 주스, 소스, 퓌레 및 Pomace 가공 중 19종 농약의 가공계수를 비교하였다. Pomace를 제외한 사과가공품은 대부분 가공계수가 1 이하의 결과를 보였다. 포도의 세척, 주스, 포도주, 건포도 및 Pomace 가공 중 21종 농약의 가공계수를 평 가하였다. 건포도의 가공계수는 대부분 1 이상으로 나타났다. 오렌지의 주스, 오일 및 마말레이드 등 가공 중 19종 농약의 가공계수를 평가한 결과, 오일의 가공계수가 1 이상으로 나타 났다. 토마토의 주스, 퓌레 및 페이스트 등 가공 중 27종 농약 의 가공계수를 비교한 결과, Pomace를 제외하고 페이스트의 가 공계수가 가장 높게 나타났다. 자두의 세척, 건조 및 퓌레 과정
Table 14 Correlation between pesticide characteristics and processing methods of strawberry

\begin{tabular}{lc}
\hline \multicolumn{1}{c}{ Pesticide characteristics and processing methods } & $\begin{array}{c}\text { Correlation } \\
\text { coefficient (n) }\end{array}$ \\
\hline Vapor pressure and jam (all samples) & $-0.251(6)$ \\
Vapor pressure and jam (formulation of EC \& SC) & - \\
Vapor pressure and jam (formulation of other) & $0.951 *(4)$ \\
Vapor pressure and jam (mode of action: systemic) & $0.931(3)$ \\
Vapor pressure and jam (mode of action: non systemic) & $-0.998^{*}(3)$ \\
\hline$*, * *$ Significant at 5 and 1\% probability levels &
\end{tabular}

중 12 종 농약의 가공계수는 건조에서 대부분 1 이상으로 나타 났다. 농약의 지용성과 휘발성 같은 물리적인 특성과 과일 가 공 중 가공계수는 사과 주스, 오렌지 주스, 토마토 퓌레와 페이 스트 및 딸기잼 가공에서 유의적인 상관관계가 있었다.

Keywords 가공계수 · 국제식품규격위원회 · 과일가공 - 잔류농 약 · 잔류농약전문가미팅

감사의 글 이 논문은 2014년도 대구대학교 학술연구비 지원에 의하여 수행 되었습니다.

\section{References}

Athanasopoulos PE, Pappas C (2000) Effects of fruit acidity and storage conditions on the rate of degradation of azinphos methyl on apples and lemons. Food Chem. 69: 69-72

Cabras P, Angioni A, Garau VL, Melis M, Pirisi FM, Cabitza F, Cubeddu M (1998a) Pesticide residues on field-sprayed apricots and in apricot drying processes. J Agric Food Chem 46: 2306-2308

Cabras P, Angioni A, Garau VL, Melis M, Pirisi FM, Cabitza F, Pala M

Table 13 Processing factor of pesticides during strawberry, cherry, mango and pineapple processing

\begin{tabular}{|c|c|c|c|c|c|c|c|c|c|c|c|}
\hline \multirow{2}{*}{ Commodity } & \multirow{2}{*}{$\begin{array}{c}\text { Pesticide } \\
\text { (Application method) }\end{array}$} & \multirow{2}{*}{ Formulation } & \multirow{2}{*}{$\begin{array}{l}\text { Mode of } \\
\text { action }\end{array}$} & \multirow{2}{*}{$\log \mathrm{K}_{\mathrm{ow}}$} & \multirow{2}{*}{$\begin{array}{l}\text { Vapor pressure } \\
(\mathrm{mPa})\end{array}$} & \multicolumn{6}{|c|}{$\mathrm{PF}^{1)}$ by Processing method } \\
\hline & & & & & & Washed & Canned & Cooked & Dried & Juice & Jam \\
\hline \multirow{7}{*}{ Strawberries } & Cycloxydim (foliar) & $\mathrm{EC}^{2)}$ & $A^{3)}$ & 1.36 & 0.01 & 1.74 & 0.9 & 0.55 & & & \\
\hline & Dimethomorph (foliar) & $\mathrm{WP}^{4)}$ & $\mathrm{S}^{5)}$ & 2.63 & $9.7 \times 10^{-4}$ & & 1.1 & & & & 0.435 \\
\hline & Fluopyram (foliar) & $\mathrm{SC}^{6)}$ & $\mathrm{N}^{7)}$ & 3.3 & $1.2 \times 10^{-3}$ & & & & & & 0.65 \\
\hline & Hexythiazox (foliar) & WP & $\mathrm{N}$ & 2.53 & $3.4 \times 10^{-3}$ & 0.485 & 0.46 & 0.49 & & & 0.665 \\
\hline & Pyraclostrobin (foliar) & $W^{8)}$ & $\mathrm{T}^{9)}$ & 3.99 & $2.6 \times 10^{-5}$ & & 0.4 & & & & 0.21 \\
\hline & Sulfoxaflor (foliar) & $\mathrm{SC}$ & $\mathrm{N}$ & 3.99 & 14 & & & & & 0.3 & 0.4 \\
\hline & Trifloxystrobin (foliar) & WG & $\mathrm{S}$ & 4.5 & $3.4 \times 10^{-3}$ & & & & & & 0.58 \\
\hline \multicolumn{6}{|c|}{ Mean } & $\begin{array}{c}1.11 \\
\pm 0.89\end{array}$ & $\begin{array}{c}0.72 \\
\pm 0.34\end{array}$ & $\begin{array}{c}0.5 \\
\pm 0.04\end{array}$ & & & $\begin{array}{c}0.49 \\
\pm 0.18\end{array}$ \\
\hline Blueberries & Fluopyram (foliar) & $\mathrm{SC}$ & $\mathrm{N}$ & 3.3 & $1.2 \times 10^{-3}$ & 0.65 & & 0.42 & & & \\
\hline \multirow{3}{*}{ Cherries } & Dithianon (foliar) & WG & $\mathrm{N}$ & 3.2 & $2.7 \times 10^{-6}$ & & N.D. & & & N.D. & N.D. \\
\hline & Pyraclostrobin (foliar) & WG & $\mathrm{T}$ & 3.99 & $2.6 \times 10^{-5}$ & & 1 & & & 0.16 & \\
\hline & Sulfoxaflor & $\mathrm{SC}$ & $\mathrm{N}$ & 3.99 & 14 & & & & 5.1 & 0.8 & 1.1 \\
\hline Mango & Thiamethoxam (foliar) & $\mathrm{SC}$ & $\mathrm{S}$ & -0.13 & $6.6 \times 10^{-6}$ & & & & 6.3 & & \\
\hline Pineapple & Fludioxonil (drench or dip) & & $\mathrm{N}$ & 4.12 & $3.9 \times 10^{-4}$ & & & & & 0.96 & \\
\hline
\end{tabular}

${ }^{1)}$ Processing factor: Residues after processing/Residues of raw material ${ }^{2)}$ Emulsifiable concentrate ${ }^{3)}$ Absorbed ${ }^{4)}$ Wettable powder ${ }^{5)}$ Systemic ${ }^{6}$ Suspension concentrate ${ }^{7}$ Non-systemic ${ }^{8)}$ Water dispersible granule ${ }^{9)}$ Translaminar 
(1998b) Pesticide residues in raisin processing. J Agric Food Chem 46: 2309-2311

Cabras P, Angioni A, Garau VL, Pirisi FM, Brandolini V, Cabitza F, Cubeddu M (1998c) Pesticide residues in prune processing. J Agric Food Chem 46: $3772-3774$

Chung HW, Ha YG, Im MH, Shin JE, Do JA, Oh JH, Cho JH, Kwon KS, Park SH (2011) Establishment of 22 pesticide MRLs in agricultural products based on risk assessment. Korean J Environ Agric 30: 166-172

FAO (2009) Submission and evaluation of pesticide residues data for the estimation of maximum residue levels in food and feed. Food and Agriculture Organization, Rome

FAO, WHO (2015) CODEX alimentarius commission procedural manual. Food and Agriculture Organization, World Health Organization, Rome

Han Y, Li W, Dong F, Xu J, Liu X, Li Y, Kong Z, Liang X, Zheng Y (2013) The behavior of chlorpyrifos and its metabolite 3,5,6-trichloro-2pyridinol in tomatoes during home canning. Food Control 31: 560-565

Im MH, Kwon KI, Park KS, Choi DM, Chang MI, Jeong JY, Lee KJ, Yun WK, Hong MK, Woo GJ (2006) Study on reduction factors of residua pesticides in processing of ginseng. The Korean $\mathrm{J}$ of pesticide Sci 10: $22-27$

Im MH, Kwon KI, Park KS, Lee KJ, Chang MI, Yun WK, Choi WJ, Yoo KS, Hong MK (2007) Reduction rate of azoxystrobin, fenhexamid and cyprodinil during ginseng processing. Korean J Food Sci Technol 39: $575-579$

JMPR (2010a) Evaluation 2010-Pesticide residues in food 2010: 15-174 (bifenthrin). Joint Meeting of the FAO Panel of Experts on Pesticide Residues, Rome

JMPR (2010b) Evaluation 2010-Pesticide residues in food 2010: 175-188 (boscalid). Joint Meeting of the FAO Panel of Experts on Pesticide Residues, Rome

JMPR (2010c) Evaluation 2010-Pesticide residues in food 2010: 223-268 (chlorantraniliprole). Joint Meeting of the FAO Panel of Experts on Pesticide Residues, Rome

JMPR (2010d) Evaluation 2010-Pesticide residues in food 2010: 269-494 (chlorothalonil). Joint Meeting of the FAO Panel of Experts on Pesticide Residues, Rome

JMPR (2010e) Evaluation 2010-Pesticide residues in food 2010: 495-765 (clothianidin). Joint Meeting of the FAO Panel of Experts on Pesticide Residues, Rome

JMPR (2010f) Evaluation 2010-Pesticide residues in food 2010: 1133-1229 (etoxazole). Joint Meeting of the FAO Panel of Experts on Pesticide Residues, Rome

JMPR (2010g) Evaluation 2010-Pesticide residues in food 2010: 1230-2365 (fenpyroximate). Joint Meeting of the FAO Panel of Experts on Pesticide Residues, Rome

JMPR (2010h) Evaluation 2010-Pesticide residues in food 2010: 1266-1392 (flubendiamide). Joint Meeting of the FAO Panel of Experts on Pesticide Residues, Rome

JMPR (2010i) Evaluation 2010-Pesticide residues in food 2010: 1415-1702 (fluopyram). Joint Meeting of the FAO Panel of Experts on Pesticide Residues, Rome

JMPR (2010j) Evaluation 2010-Pesticide residues in food 2010: 1737-1778 (novaluron). Joint Meeting of the FAO Panel of Experts on Pesticide Residues, Rome

JMPR (2011a) Evaluation 2011-Pesticide residues in food 2011: 231-404 (emamectin benzoate). Joint Meeting of the FAO Panel of Experts on Pesticide Residues, Rome

JMPR (2011b) Evaluation 2011-Pesticide residues in food 2011: 405-500 (etofenprox). Joint Meeting of the FAO Panel of Experts on Pesticide Residues, Rome

JMPR (2011c) Evaluation 2011-Pesticide residues in food 2011: 501-618 (flutriafol). Joint Meeting of the FAO Panel of Experts on Pesticide Residues, Rome

JMPR (2011d) Evaluation 2011-Pesticide residues in food 2011: 710-726 (hexythiazox). Joint Meeting of the FAO Panel of Experts on Pesticide
Residues, Rome

JMPR (2011e) Evaluation 2011-Pesticide residues in food 2011: 905-1088 (pyraclostrobin). Joint Meeting of the FAO Panel of Experts on Pesticide Residues, Rome

JMPR (2011f) Evaluation 2011-Pesticide residues in food 2011: 1271-1418 (sulfoxaflor). Joint Meeting of the FAO Panel of Experts on Pesticide Residues, Rome

JMPR (2011g) Evaluation 2011-Pesticide residues in food 2011: 1419-1614 (tebuconazole). Joint Meeting of the FAO Panel of Experts on Pesticide Residues, Rome

JMPR (2012a) Evaluation 2012-Pesticide residues in food 2012: 1-152 (ametoctradin). Joint Meeting of the FAO Panel of Experts on Pesticide Residues, Rome

JMPR (2012b) Evaluation 2012-Pesticide residues in food 2012: 181-250 (chlorfenapyr). Joint Meeting of the FAO Panel of Experts on Pesticide Residues, Rome

JMPR (2012c) Evaluation 2012-Pesticide residues in food 2012: 260-384 (cycloxydim). Joint Meeting of the FAO Panel of Experts on Pesticide Residues, Rome

JMPR (2012d) Evaluation 2012-Pesticide residues in food 2012: 385-402 (cyfluthrin/beta-cyfluthrin). Joint Meeting of the FAO Panel of Experts on Pesticide Residues, Rome

JMPR (2012e) Evaluation 2012-Pesticide residues in food 2012: 447-590 (dinotefuran). Joint Meeting of the FAO Panel of Experts on Pesticide Residues, Rome

JMPR (2012f) Evaluation 2012-Pesticide residues in food 2012: 603-616 (fludioxonil). Joint Meeting of the FAO Panel of Experts on Pesticide Residues, Rome

JMPR (2012g) Evaluation 2012-Pesticide residues in food 2012: 617-658 (fluopyram). Joint Meeting of the FAO Panel of Experts on Pesticide Residues, Rome

JMPR (2012h) Evaluation 2012-Pesticide residues in food 2012: 659-936 (fluxapyroxad). Joint Meeting of the FAO Panel of Experts on Pesticide Residues, Rome

JMPR (2012i) Evaluation 2012-Pesticide residues in food 2012: 937-1182 (glufosinate ammonium). Joint Meeting of the FAO Panel of Experts on Pesticide Residues, Rome

JMPR (2012j) Evaluation 2012-Pesticide residues in food 2012: 1337-1364 (methoxyfenozide). Joint Meeting of the FAO Panel of Experts on Pesticide Residues, Rome

JMPR (2012k) Evaluation 2012-Pesticide residues in food 2012: 1365-1652 (penthiopyrad). Joint Meeting of the FAO Panel of Experts on Pesticide Residues, Rome

JMPR (20121) Evaluation 2012-Pesticide residues in food 2012: 2037-2057 (trifloxystrobin). Joint Meeting of the FAO Panel of Experts on Pesticide Residues, Rome

JMPR (2013a) Evaluation 2013-Pesticide residues in food 2013: 361-612 (cyantraniliprole). Joint Meeting of the FAO Panel of Experts on Pesticide Residues, Rome

JMPR (2013b) Evaluation 2013-Pesticide residues in food 2013: 615-658 (cyprodinil). Joint Meeting of the FAO Panel of Experts on Pesticide Residues, Rome

JMPR (2013c) Evaluation 2013-Pesticide residues in food 2013: 673-724 (difenoconazole). Joint Meeting of the FAO Panel of Experts on Pesticide Residues, Rome

JMPR (2013d) Evaluation 2013-Pesticide residues in food 2013: 803-910 (dithianon). Joint Meeting of the FAO Panel of Experts on Pesticide Residues, Rome

JMPR (2013e) Evaluation 2013-Pesticide residues in food 2013: 911-914 (fenbuconazole). Joint Meeting of the FAO Panel of Experts on Pesticide Residues, Rome

JMPR (2013f) Evaluation 2013-Pesticide residues in food 2013: 961-970 (fludioxonil). Joint Meeting of the FAO Panel of Experts on Pesticide Residues, Rome

JMPR (2013g) Evaluation 2013-Pesticide residues in food 2013: 1361-1368 
(propiconazole). Joint Meeting of the FAO Panel of Experts on Pesticide Residues, Rome

JMPR (2013h) Evaluation 2013-Pesticide residues in food 2013: 1369-1384 (pyrimethani). Joint Meeting of the FAO Panel of Experts on Pesticide Residues, Rome

JMPR (2013i) Evaluation 2013-Pesticide residues in food 2013: 1411-1498 (tolfenpyrad). Joint Meeting of the FAO Panel of Experts on Pesticide Residues, Rome

JMPR (2013j) Evaluation 2013-Pesticide residues in food 2013: 1507-1594 (triflumizole). Joint Meeting of the FAO Panel of Experts on Pesticide Residues, Rome

JMPR (2014a) Evaluation 2014-Pesticide residues in food 2014: 175-280 (cyflumetofen). Joint Meeting of the FAO Panel of Experts on Pesticide Residues, Rome

JMPR (2014b) Evaluation 2014-Pesticide residues in food 2014: 281-340 (dichlobenil). Joint Meeting of the FAO Panel of Experts on Pesticide Residues, Rome

JMPR (2014c) Evaluation 2014-Pesticide residues in food 2014: 341-406 (dimethomorph). Joint Meeting of the FAO Panel of Experts on Pesticide Residues, Rome

JMPR (2014d) Evaluation 2014-Pesticide residues in food 2014: 445-600 (fenamidone). Joint Meeting of the FAO Panel of Experts on Pesticide Residues, Rome

JMPR (2014e) Evaluation 2014-Pesticide residues in food 2014: 601-714 (fenpropathrin). Joint Meeting of the FAO Panel of Experts on Pesticide Residues, Rome

JMPR (2014f) Evaluation 2014-Pesticide residues in food 2014: 717-798 (fluensulfone). Joint Meeting of the FAO Panel of Experts on Pesticide Residues, Rome

JMPR (2014g) Evaluation 2014-Pesticide residues in food 2014: 857-928 (fluopyram). Joint Meeting of the FAO Panel of Experts on Pesticide Residues, Rome

JMPR (2014h) Evaluation 2014-Pesticide residues in food 2014: 1179-1296 (metrafenone). Joint Meeting of the FAO Panel of Experts on Pesticide Residues, Rome

JMPR (2014i) Evaluation 2014-Pesticide residues in food 2014: 1297-1472 (myclobutanil). Joint Meeting of the FAO Panel of Experts on Pesticide Residues, Rome

JMPR (2014j) Evaluation 2014-Pesticide residues in food 2014: 1477-1520 (propamocarb). Joint Meeting of the FAO Panel of Experts on Pesticide Residues, Rome

JMPR (2014k) Evaluation 2014-Pesticide residues in food 2014: 1815-1856 (thiamethoxam). Joint Meeting of the FAO Panel of Experts on Pesticide Residues, Rome

JMPR (2014l) Evaluation 2014-Pesticide residues in food 2014: 1857-1953 (triforine). Joint Meeting of the FAO Panel of Experts on Pesticide Residues, Rome
Kaushik G, Satya S, Naik SN (2009) Food processing a tool to pesticide residue dissipation - A review. Food Research Int'l 42: 26-40

Keikotlhaile BM, Spanoghe P, Steurbaut W (2010) Effects of food processing on pesticide residues in fruits and vegetables: A meta-analysis approach. Food and Chem Toxicology 48: 1-6

Kim JG, Kim SS, Park HR, Ji KY, Lee KH, Ham HJ, Im MH, Hur JH (2009) Residues of azoxystrobin during cultivation and processing of ginseng. The Korean J of Pesticide Sci 13: 232-240

Lee EY, Noh HH, Park YS, Kang KW, Lee KH, Park HK, Kwon CH, Im MH, Kyung KS (2009) Processing and reduction factors of pesticide residues in chinese matrimony vine and jujube by drying. The Korean $\mathrm{J}$ of Pesticide Sci 13: 159-164

Lee JK, Woo HD (2010) Current status for management of pesticide maximum residue limits in foods. Food Sci and Industry 43: 2-23

Lee JY, Noh HH, Lee KH, Park HK, Oh JH, Im MH, Kwon CH, Lee JK, Woo HD, Kwon KS, Kyung KS (2012) Processing factors of azoxystrobin in processed ginseng products. The Korean $\mathrm{J}$ of Pesticide Sci 16: 222-229

Lentza-Rizos C (1995) Residues of iprodione in fresh and canned peaches after pre- and postharvest treatment. J Agric Food Chem 43: 1357-1360

Lentza-Rizos C, Avramides EJ, Kokkinaki K (2006) Residues of azoxystrobin from grapes to raisins. J Agric Food Chem 54: 138-141

Li M, Liu Y, Fan B, Lu J, He Y, Kong Z, Zhu Y, Jian Q, Wang F (2015) A chemometric processing-factor-based approach to the determination of the fates of five pesticides during apple processing. Food Sci and Technol 63: 1102-1109

MFDS (2015a) Food standard and specification. Ministry of Food and Drug Safety, Cheongju. 35-36

MFDS (2015b) Food standard and specification. Ministry of Food and Drug Safety, Cheongju. 494-503

Noh HH, Lee JY, Park SH, Lee KH, Oh JH, Im MH, Kyung KS (2012b) Residual characteristics of azoxystrobin and difenoconazole in ginseng. The Korean J of Pesticide Sci 16: 131-136

Noh HH, Lee KH, Lee JY, Lee EY, Park YS, Park HK, Oh JH, Im MH, Lee YJ, Baeg IH, Kyung KS (2012a) Residual characteristics and processing factors of difenoconazole in Fresh ginseng and processed ginseng products. The Korean J of Pesticide Sci 16: 35-42

Park SY, Kang HR, Ko KY, Gil KH, Im MH, Lee KS (2009a) On the processing and reduction factors of several pesticides with welsh onion. The Korean J of Pesticide Sci 13: 249-255

Park SY, Park KS, Im MH, Choi H, Chang MI, Kwon CH, Kim SG, Lee HK, Hong MK, Shim JH, Kim JH (2009b) Studies for the processing factors of pesticides during the milling of wheat grain. The Korean J of Pesticide Sci Science 13: 70-78

Zabik MJ, El-Hadidi MFA, Cash JN, Zabik ME, Jones AL (2000) Reduction of azinphos-methyl, chlorpyrifos, esfenvalerate, and methomyl residues in processed apples. J Agric Food Chem 48: 4199-4203 